\title{
Quasi-Cyclic LDPC Codes based on Pre-Lifted Protographs
}

\author{
David G. M. Mitchell, Member, IEEE, Roxana Smarandache, Member, IEEE, \\ and Daniel J. Costello, Jr., Life Fellow, IEEE
}

\begin{abstract}
Quasi-cyclic low-density parity-check (QC-LDPC) codes based on protographs are of great interest to code designers because analysis and implementation are facilitated by the protograph structure and the use of circulant permutation matrices for protograph lifting. However, these restrictions impose undesirable fixed upper limits on important code parameters, such as minimum distance and girth. In this paper, we consider an approach to constructing QC-LDPC codes that uses a two-step lifting procedure based on a protograph, and, by following this method instead of the usual one-step procedure, we obtain improved minimum distance and girth properties. We also present two new design rules for constructing good QC-LDPC codes using this two-step lifting procedure, and in each case we obtain a significant increase in minimum distance and achieve a certain guaranteed girth compared to one-step circulant-based liftings. The expected performance improvement is verified by simulation results.
\end{abstract}

Index Terms-Low-density parity-check (LDPC) codes, girth, minimum distance, protograph, quasi-cyclic codes, Tanner graph.

\section{INTRODUCTION}

A protograph [1] is a small Tanner graph [2] described by an $n_{c} \times n_{v}$ biadjacency matrix $\mathbf{B}$, known as a base matrix, that consists of non-negative integers $B_{i, j}$ that correspond to $B_{i, j}$ parallel edges in the graph. A protograph-based code is obtained by taking an $N$-fold graph cover [3], or "lifting", of a given protograph and can be described by an $N n_{c} \times N n_{v}$ parity-check matrix obtained by replacing each non-zero entry $B_{i, j}$ by a sum of $B_{i, j}$ non-overlapping permutation matrices of size $N \times N$ and each zero entry by an $N \times N$ all-zero matrix. The set of all such codes that can be derived from the protograph in this fashion is referred to as a code ensemble. Low-density parity-check (LDPC) code ensembles [4] based on a protograph form a subclass of multi-edge type codes [5] that, for suitably-designed protographs, have many desirable features, such as good iterative decoding thresholds and linear

This work was supported in part by the National Science Foundation under Grant Numbers CCF-1161754, CCF-1252788, and DMS-1313221. The material in this paper was presented in part at the IEEE Information Theory Workshop, Paraty, Brazil, October 2011, and in part at the IEEE Information Theory Workshop, Lausanne, Switzerland, September 2012.

D. G. M. Mitchell, R. Smarandache, and D. J. Costello, Jr. are with the Department of Electrical Engineering, University of Notre Dame, Notre Dame, IN 46556, USA (e-mail: david.mitchell@nd.edu; rsmarand@nd.edu; costello.2@nd.edu). D. G. M. Mitchell and R. Smarandache are also with the Department of Mathematics, University of Notre Dame, Notre Dame, IN 46556, USA.

Copyright (c) 2014 IEEE. Personal use of this material is permitted. However, permission to use this material for any other purposes must be obtained from the IEEE by sending a request to pubs-permissions@ieee.org. minimum distance growth, i.e., they are asymptotically good (see, e.g., [6]-[8]).

The construction of quasi-cyclic LDPC (QC-LDPC) codes [9]-[22] can be seen as a special case of the protographbased construction in which the $N$-fold cover is obtained by restricting the edge permutations to be cyclic and can be described by an $N n_{c} \times N n_{v}$ parity-check matrix formed as an $n_{c} \times n_{v}$ array of $N \times N$ circulant matrices. Members of a protograph-based LDPC code ensemble that are QC are particularly attractive from an implementation standpoint, since they can be encoded with low complexity using simple feedback shift-registers [12], [14] and their structure leads to efficiencies in decoder design [23], [24]. Moreover, QC-LDPC codes can be shown to perform well compared to random LDPC codes for moderate block lengths [9], [16], [19], [20]. However, unlike typical members of an asymptotically good protograph-based LDPC code ensemble, the QC sub-ensemble does not have linear distance growth. Indeed, if the protograph base matrix consists of only ones and zeros, then the minimum Hamming distance is bounded above by $\left(n_{c}+1\right)$ !, where $n_{c}$ is the number of check nodes in the protograph, regardless of the lifting factor $N$ [10], [25].

A great deal of research effort has been devoted to designing QC-LDPC codes with large girth (see, e.g., [10], [13], [22], [26]) and minimum distance (see, e.g., [9], [10], [16][21]). QC-LDPC codes based on protographs have also been designed to improve certain characteristics, such as girth [27][29] or lowering the "error floor" [30]. In [31], lower bounds on the size of the necessary lifting factor $N$ of a protograph required to achieve a certain girth is investigated for $\mathrm{QC}$ LDPC codes derived from several simple protograph types. Minimum distance bounds for protograph-based QC-LDPC codes were presented in [21] and later improved for several cases in [32]. A useful feature of the approach presented in the current paper, which we will demonstrate later, is that a good existing QC-LDPC code design can be used in conjunction with our methodology to improve code performance.

Several authors have also considered two- or multi-step liftings of a base graph. In [33], irregular protograph-based QC-LDPC codes are proposed with parallel edges in the protograph. In order to have only single edges in the code's Tanner graph, which is desirable for efficient implementation, the authors first employ an $m$-step expansion, where $m$ is sufficiently large to disperse the parallel edges, before applying a second lifting step. In [21], the authors present an example showing that a QC code obtained from a double lifting has a larger minimum distance than a single lifted code. Also, so- 
called hierarchical QC-LDPC codes have been constructed in a recent paper [34]. These high-girth constructions are obtained by taking repeated circulant-based liftings of a base graph, but the authors do not consider minimum distance.

In this paper, we investigate QC-LDPC codes that are constructed using a two-step lifting procedure based on a protograph: a "pre-lifting" step where we take an $m$-fold graph cover of the protograph, where $m$ is typically small, and a "second-lifting" step where we take an $r$-fold graph cover of the pre-lifted protograph, where $r$ is typically large and the permutations are chosen to be cyclic 1 As a result of the prelifting, we can construct QC-LDPC codes with increased girth and minimum distance while maintaining the circulant-based structure that facilitates efficient implementation. In particular, we show that the QC-LDPC code ensemble obtained from a pre-lifted protograph can have an increased upper bound on minimum distance compared to the QC-LDPC code ensemble obtained from the original protograph and we demonstrate the existence of codes with minimum distance exceeding the original bound. We also present two design rules for code construction: one uses only commuting pairs of permutation matrices at the first (pre-lifting) stage, while the other uses at least one pair of non-commuting permutation matrices. In each case, we obtain a significant increase in the minimum distance and achieve a certain guaranteed girth compared to a one-step circulant-based lifting of the original protograph. The expected performance improvement is verified by simulation results.

The paper is structured as follows. In Section II we provide the necessary background material, describe the structure of the QC sub-ensemble of a protograph-based LDPC code ensemble, and review existing bounds concerning the minimum Hamming distance of QC-LDPC protograph-based codes. In Section III, we introduce the concept of pre-lifting, discuss some necessary conditions to permit increased minimum distance and girth for our construction technique, and present two new code design rules. In Section IV we focus on the prelifting step and derive circulant-based codes with minimum distance and girth exceeding the original bounds for QC codes without pre-lifting. Sections $\mathrm{V}$ and VI demonstrate the application of the two code design rules. The expected performance improvement is verified by simulation results. In Section VII we construct a nested family of QC-LDPC codes with design rates $R=1 / 4,2 / 5,1 / 2$, and $4 / 7$ and robustly good performance by applying the pre-lifting technique to a QC-LDPC code with large girth taken from the literature. Finally, concluding remarks are given in Section VIII.

\section{BASIC DEFINITIONS, NOTATION, AND BACKGROUND}

\section{Notation}

- For any positive integer $L,[L] \triangleq\{0,1, \ldots, L-1\}$.

- $\mathbb{Z}$ is the ring of integers; $\mathbb{F}_{2}$ is the Galois field of size 2 .

- $\mathbb{F}_{2}^{n}$ and $\mathbb{F}_{2}^{k \times n}$ are, respectively, the set of row vectors over $\mathbb{F}_{2}$ of length $n$ and the set of matrices over $\mathbb{F}_{2}$ of size $k \times n$.

\footnotetext{
${ }^{1}$ It is also possible to construct QC-LDPC codes in this way using more than two lifting steps, but only two-step liftings are considered in this paper.
}

\section{Linear codes}

- All the codes in this paper are binary linear codes.

- An $\left[n, k, d_{\text {min }}\right]$ linear code $\mathcal{C}$ of length $n$, dimension $k$, and minimum Hamming distance $d_{\text {min }}$ can be specified as the null space of a $p \times n$ (scalar) parity-check matrix $\mathbf{H} \in \mathbb{F}_{2}^{p \times n}$, where the rank of the matrix is $n-k \leq p$, i.e.,

$$
\mathcal{C}=\left\{\mathbf{c} \in \mathbb{F}_{2}^{n} \mid \mathbf{H} \cdot \mathbf{c}^{\top}=\mathbf{0}^{\top}\right\}
$$

where ${ }^{\mathrm{T}}$ denotes transposition.

\section{Tanner graphs}

- With a parity-check matrix $\mathbf{H}$ we associate a bipartite Tanner graph [2] in the usual way.

- The girth of a Tanner graph associated with a paritycheck matrix $\mathbf{H}$ is the length of the shortest cycle in the graph and is denoted by $g$.

\section{Permutations}

- An $N$-permutation $\sigma$ is a one-to-one function on the set $\mathcal{N}=\{1,2, \ldots, N\}$ described as:

$$
\sigma \triangleq\left(\begin{array}{cccc}
1 & 2 & \cdots & N \\
\sigma(1) & \sigma(2) & \cdots & \sigma(N)
\end{array}\right) .
$$

- Any permutation $\sigma$ can be represented by an $N \times N$ permutation matrix $\mathbf{P}$, where $\mathbf{P}$ has all entries equal to zero except for $N$ entries equal to one at the positions $(i, \sigma(i))$ for all $i \in \mathcal{N}$.

- Composing two permutations $\sigma$ and $\tau$ on $\mathcal{N}$ gives two new permutations, $\sigma \tau$ and $\tau \sigma$, which in general are not equal. Equivalently, the product of two permutation matrices $\mathbf{P}$ and $\mathbf{Q}$ gives two new permutation matrices PQ and QP, which in general are not equal.

- (Permutation) matrices $\mathbf{P}$ and $\mathbf{Q}$ are said to have an overlapping column (or row) if $\mathbf{P}$ and $\mathbf{Q}$ have at least one identical column (or row). Further, $\mathbf{P}$ and $\mathbf{Q}$ are said to be overlapping if they have at least one overlapping column (or row), or non-overlapping if they have no overlapping columns (or rows).

- Matrix $\mathbf{P}$ is said to have a fixed column (or row) if it overlaps with the identity matrix in at least one column (or row), or equivalently $\sigma$ has a fixed point if $\sigma(i)=i$ for some $i \in \mathcal{N}$.

- Two matrices $\mathbf{P}$ and $\mathbf{Q}$ commute if $\mathbf{P Q}=\mathbf{Q P}$.

- Two (permutation) matrices $\mathbf{P}$ and $\mathbf{Q}$ are said to be strongly noncommutative if $\mathrm{PQ}$ and $\mathrm{QP}$ have no overlapping columns, i.e., each column in $\mathbf{P Q}$ differs from the corresponding column in QP.

\section{Circulant and circulant-block permutations}

- The notation $\mathbf{I}_{a}^{N}$ is used to denote the $N \times N$ identity matrix with each row cyclically shifted to the left by $a$ positions. This matrix, and its corresponding permutation $\sigma$, will be referred to as a circulant permutation matrix or permutation, respectively.

- Let $a, b, m \in \mathbb{Z}, a, b \geq 0, m \geq 1$. Then

Property 1. The circulant permutation matrix $\mathbf{I}_{a}^{m}$ has a fixed column iff $a \equiv 0 \bmod m$. If $\mathbf{I}_{a}^{m}$ has a fixed column, then $\mathbf{I}_{a}^{m}=\mathbf{I}_{0}^{m}$. 
Property 2. The product of two circulant permutation matrices $\mathbf{I}_{a}^{m}$ and $\mathbf{I}_{b}^{m}$ is given by $\mathbf{I}_{a}^{m} \mathbf{I}_{b}^{m}=\mathbf{I}_{(a+b)}^{m} \bmod m$. Property 3. The transposition of a circulant permutation matrix $\mathbf{I}_{a}^{m}$ is $\left(\mathbf{I}_{a}^{m}\right)^{\top}=\mathbf{I}_{(m-a)}^{m} \bmod m$.

- By Property 2, any two circulant permutation matrices commute. This is not true for permutation matrices in general.

- We define an $m r \times m r$ circulant-block permutation matrix $\mathbf{C}$ as an $m \times m$ array of $r \times r$ circulant permutation matrices and all-zero matrices arranged such that each (block) row and column contains precisely one circulant permutation matrix, i.e.,

$$
\mathbf{C}=\operatorname{diag}\left(\mathbf{I}_{s_{1}}^{r}, \mathbf{I}_{s_{2}}^{r}, \ldots, \mathbf{I}_{s_{m}}^{r}\right) \cdot \tilde{\mathbf{P}},
$$

where $m$ and $r$ are positive integers, $s_{k} \in[r], k \in$ $\{1,2, \ldots, m\}$, are called the shift parameters, $\tilde{\mathbf{P}} \triangleq \mathbf{P} \otimes \mathbf{I}_{0}^{r}$ denotes the Kronecker product of an $m \times m$ permutation matrix $\mathbf{P}$ and $\mathbf{I}_{0}^{r}$, and, in a slight abuse of notation,

$$
\begin{aligned}
& \operatorname{diag}\left(\mathbf{I}_{s_{1}}^{r}, \mathbf{I}_{s_{2}}^{r}, \ldots, \mathbf{I}_{s_{m}}^{r}\right)= \\
& {\left[\begin{array}{cccc}
\mathbf{I}_{s_{1}}^{r} & \mathbf{0} & \cdots & \mathbf{0} \\
\mathbf{0} & \mathbf{I}_{s_{2}}^{r} & \cdots & \mathbf{0} \\
\vdots & \vdots & \ddots & \vdots \\
\mathbf{0} & \mathbf{0} & \cdots & \mathbf{I}_{s_{m}}^{r}
\end{array}\right]_{m r \times m r}}
\end{aligned}
$$

The corresponding permutation $\sigma$ will be referred to as a circulant-block permutation.

- An example of an $m r \times m r$ circulant-block permutation matrix $\mathbf{C}$ with $m=4, r=7$, shift parameters $\left(s_{1}, s_{2}, s_{3}, s_{4}\right)=(1,4,2,5)$, and $m \times m$ permutation matrix

$$
\mathbf{P}=\left[\begin{array}{llll}
1 & 0 & 0 & 0 \\
0 & 0 & 0 & 1 \\
0 & 1 & 0 & 0 \\
0 & 0 & 1 & 0
\end{array}\right]_{4 \times 4}
$$

is

$$
\mathbf{C}=\left[\begin{array}{cccc}
\mathbf{I}_{1}^{7} & \mathbf{0} & \mathbf{0} & \mathbf{0} \\
\mathbf{0} & \mathbf{0} & \mathbf{0} & \mathbf{I}_{4}^{7} \\
\mathbf{0} & \mathbf{I}_{2}^{7} & \mathbf{0} & \mathbf{0} \\
\mathbf{0} & \mathbf{0} & \mathbf{I}_{5}^{7} & \mathbf{0}
\end{array}\right]_{21 \times 21}
$$

\section{Protograph-based LDPC codes}

- A protograph [1] is a small bipartite graph, represented by a parity-check or base biadjacency matrix B (as described in Section \).

- The parity-check matrix $\mathbf{H}$ of a protograph-based LDPC block code is created by replacing each non-zero entry $B_{i, j}$ in $\mathbf{B}$ by a sum of $B_{i, j}$ non-overlapping permutation matrices of size $N \times N$ and each zero entry by the $N \times N$ all-zero matrix, where $B_{i, j}$ is a non-negative integer.

- A parity-check matrix $\mathbf{H}$ that has been created from $\mathbf{B}$ using the protograph construction method with $N \times N$ permutation matrices is denoted by

$$
\mathbf{H}=\mathbf{B}^{\uparrow N} \text {. }
$$

- Graphically, this operation is equivalent to taking an $N$ fold graph cover [3], or " $N$-lift", of the protograph.
- An example of the lifting procedure applied to a $(3,4)$ regular protograph is shown in Fig. 1. It is an important feature of this construction that each lifted code inherits the degree distribution and local graph neigbourhood structure of the protograph.
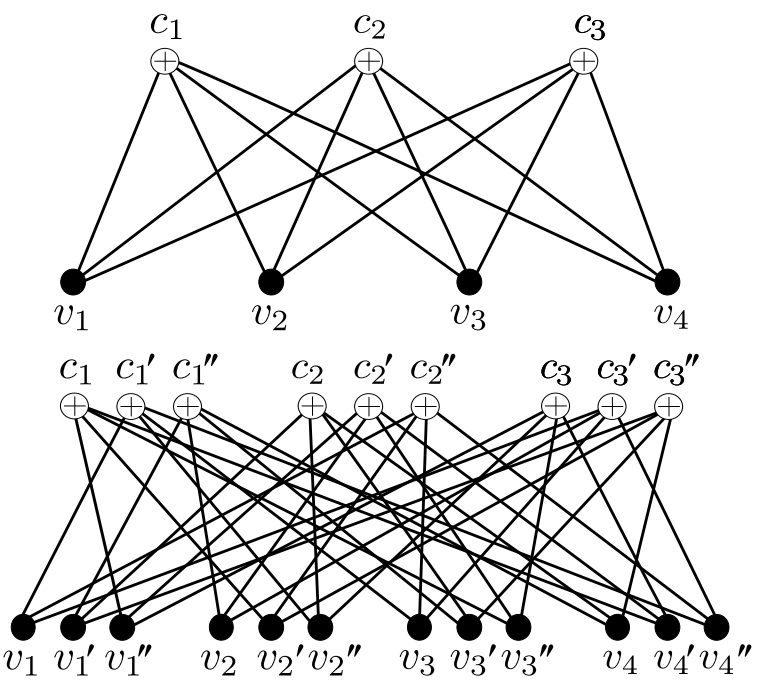

Fig. 1. Tanner graphs of a (3,4)-regular protograph (top) and a (3, 4)regular QC protograph-based code obtained from the protograph with $N=3$ (bottom).

- The ensemble of protograph-based LDPC codes with block length $n=N n_{v}$, denoted $\xi_{\mathbf{B}}(N)$, is defined as the set of matrices $\mathbf{H}$ that can be derived from a given base matrix B using all possible combinations of $N \times N$ permutation matrices.

- The most general case of an LDPC code lifted from an $n_{c} \times n_{v}$ all-one base matrix is given by a paritycheck matrix $\mathbf{H}=\mathbf{B}^{\uparrow N}$ consisting of an $n_{c} \times n_{v}$ array of permutation matrices $\mathbf{Q}_{i, j}, i \in\left\{1,2, \ldots, n_{c}\right\}$, $j \in\left\{1,2, \ldots, n_{v}\right\}$. Without loss of generality, after row and column permutations, any $n_{c} \times n_{v}$ all-one base matrix can be written as

$$
\left[\begin{array}{cccc}
\mathbf{I}_{0}^{N} & \mathbf{I}_{0}^{N} & \ldots & \mathbf{I}_{0}^{N} \\
\mathbf{I}_{0}^{N} & \mathbf{P}_{2,2} & \ldots & \mathbf{P}_{2, n_{v}} \\
\vdots & \vdots & \ldots & \vdots \\
\mathbf{I}_{0}^{N} & \mathbf{P}_{n_{c}, 2} & \ldots & \mathbf{P}_{n_{c}, n_{v}}
\end{array}\right]
$$

where $\mathbf{P}_{i, j}$ is a permutation matrix, $i \in\left\{2,3, \ldots, n_{c}\right\}$, $j \in\left\{2,3, \ldots, n_{v}\right\}, \mathbf{I}_{0}^{N}$ is the identity matrix, and all matrices are of size $N$. The minimum distance and girth of the code and graph, respectively, are not affected by such operations. If all the permutation matrices $\mathbf{Q}_{i, j}$ are chosen to be circulant (or circulant-block, as defined above), then the resulting permutation matrices $\mathbf{P}_{i, j}$ in (2) are also circulant (resp. circulant-block). See Appendix $\mathrm{A}$ for details.

$Q C$ sub-ensembles

- The $Q C$ sub-ensemble of $\xi_{\mathbf{B}}(N)$, denoted $\xi_{\mathbf{B}}^{Q C}(N)$, is the subset of parity-check matrices in $\xi_{\mathbf{B}}(N)$ where all the permutation matrices are chosen to be circulant. 
- We denote a parity-check matrix $\mathbf{H}$ that has been $N$-lifted from B using only circulant permutation matrices as

$$
\mathbf{H}=\mathbf{B}^{\circlearrowleft N} \text {. }
$$

- The codes that are constructed using this technique are QC with period $n_{v}$, i.e., cyclically shifting the $N$ symbols in each of the $n_{v}$ blocks in a codeword by one position results in a codeword 2

- By restricting the choice of permutation matrices to come from the circulant subset $\left\{\mathbf{I}_{a}^{N} \mid a \in[N]\right\}$, the resulting parity-check matrix $\mathbf{H}$ is the parity-check matrix of a QC-LDPC code, i.e.,

$$
\mathbf{H}=\mathbf{B}^{\circlearrowleft N} \in \xi_{\mathbf{B}}^{Q C}(N) \subseteq \xi_{\mathbf{B}}(N) .
$$

In graphical terms, we refer to this operation as a "circulant-based lifting".

- Note that the sub-ensemble $\xi_{\mathrm{B}}^{Q C}(N)$ is smaller than the ensemble $\xi_{\mathbf{B}}(N)$. This follows since there are only $N$ out of $N$ ! permutations that are circulant, i.e., the fraction of choices of permutation matrices that are circulant is $N / N !=1 /(N-1)$ !, which tends to zero as $N \rightarrow \infty$. It follows that, if the base matrix $\mathbf{B}$ contains only ones and zeros, the fraction of codes in the ensemble that are composed of circulant matrices is $(1 /(N-1) !)^{t}$, where $t$ is the number of ones in $\mathbf{B}$. Parallel edges in $\mathbf{B}$ further reduce this fraction. Consequently, asymptotic ensemble average results, such as those reported in [6]-[8], cannot be used to describe the behavior of this sub-ensemble, since the members are not typical, i.e., the probability of picking such a code vanishes in the limit of large $N$, so we cannot say the codes perform close to the ensemble mean.

QC-code examples

Example 1. The $3 \times 4$ all-ones base matrix

$$
\mathbf{B}=\left[\begin{array}{llll}
1 & 1 & 1 & 1 \\
1 & 1 & 1 & 1 \\
1 & 1 & 1 & 1
\end{array}\right]
$$

can be lifted using circulant permutations with lifting factor $N=3$ to form the following $(3,4)$-regular QC-LDPC code with length $n=12$ and parity-check matrix

$$
\mathbf{H}=\mathbf{B}^{\circlearrowleft 3}=\left[\begin{array}{cccc}
\mathbf{I}_{1}^{3} & \mathbf{I}_{2}^{3} & \mathbf{I}_{1}^{3} & \mathbf{I}_{2}^{3} \\
\mathbf{I}_{2}^{3} & \mathbf{I}_{1}^{3} & \mathbf{I}_{2}^{3} & \mathbf{I}_{0}^{3} \\
\mathbf{I}_{1}^{3} & \mathbf{I}_{1}^{3} & \mathbf{I}_{1}^{3} & \mathbf{I}_{2}^{3}
\end{array}\right] \in \xi_{\mathbf{B}}^{Q C}(3) .
$$

The corresponding Tanner graphs are shown in Fig. 1

Example 2. The (3,4)-regular QC-LDPC Tanner code (see Example 11 in [21]) has a parity-check matrix, lifted from the $3 \times 4$ all-ones base matrix $\mathbf{B}$, given by

$$
\mathbf{H}=\mathbf{B}^{\circlearrowleft N}=\left[\begin{array}{cccc}
\mathbf{I}_{1}^{N} & \mathbf{I}_{2}^{N} & \mathbf{I}_{4}^{N} & \mathbf{I}_{8}^{N} \\
\mathbf{I}_{5}^{N} & \mathbf{I}_{10}^{N} & \mathbf{I}_{20}^{N} & \mathbf{I}_{9}^{N} \\
\mathbf{I}_{25}^{N} & \mathbf{I}_{19}^{N} & \mathbf{I}_{7}^{N} & \mathbf{I}_{14}^{N}
\end{array}\right] \in \xi_{\mathbf{B}}^{Q C}(N) .
$$

\footnotetext{
${ }^{2}$ Strictly speaking, for the code to be QC with period $n_{v}$, it must satisfy the property that, for each codeword, a cyclic shift of $n_{v}$ positions results in a codeword. This requires the columns of $\mathbf{H}$ to be reordered accordingly.
}

for the lifting factor $N=31$, this parity-check matrix defines a $[124,33,24]$ code with girth 8 .

\section{Minimum distance bounds for $Q C$ sub-ensembles}

- If the base matrix B contains only ones and zeros, it is well known that the minimum distance of any code from the QC sub-ensemble of protograph-based LDPC codes can immediately be bounded above by $\left(n_{c}+1\right)$ ! [10], [25].

Theorem 1 If a parity-check matrix of height $n_{c} M$ contains a submatrix of height $n_{c} M$ and width $\left(n_{c}+1\right) M$ containing a grid of $n_{c}\left(n_{c}+1\right)$ permutation matrices that all commute with each other, then the corresponding code has minimum distance less than or equal to $\left(n_{c}+1\right)$ !.

- In [21], the authors provide an improved bound that, in addition to giving tighter bounds for base matrices with only zero and one entries, can also be applied to base matrices with entries larger than one, i.e., protographs with parallel edges. Let the permanent of an $m \times m$ matrix $\mathbf{M}$ be defined as

$$
\operatorname{perm}(\mathbf{M})=\sum_{\sigma} \prod_{x=1}^{m} M_{x, \sigma(x)},
$$

where $M_{x, \sigma(x)}$ is the entry in $\mathbf{M}$ at position $(x, \sigma(x))$ and we sum over the $m$ ! permutations $\sigma$ of the set $\{1,2, \ldots, m\}$. Then the minimum distance of a code drawn from the QC sub-ensemble of a protograph-based ensemble can be bounded above as follows.

Theorem 2 Let $\mathcal{C}$ be a code in $\xi_{\mathrm{B}}^{Q C}(N)$, the $Q C$ sub-ensemble of the protograph-based ensemble of codes formed from a base matrix $\mathbf{B}$. Then the minimum Hamming distance of $C$ is bounded above as

$$
d_{\min } \leq \min _{\substack{S \subseteq\left\{1,2, \ldots, n_{v}\right\} \\|S|=n_{c}+1}} \sum_{i \in S} \operatorname{perm}\left(\mathbf{B}_{S \backslash i}\right),
$$

where perm $\left(\mathbf{B}_{S \backslash i}\right)$ denotes the permanent of the matrix consisting of the $n_{c}$ columns of $\mathbf{B}$ in the set $S \backslash i$ and the $\min ^{*}\{\cdot\}$ operator returns the smallest non-zero value from a set.

- For all the protographs considered in this paper, the bound on minimum distance obtained using (6) is at least as tight as $\left(n_{c}+1\right)$ !, and in many cases it is tighter. Recently, Butler and Siegel further improved this bound for protographs with irregular structures and punctured symbols [32].

\section{Girth results for $Q C$ sub-ensembles}

In this paper, our primary goal is to construct protographbased QC-LDPC codes with large minimum distance; however, when using (sub-optimal) iterative decoding techniques, such as belief propagation (BP) decoding, graph-based properties, such as short cycles in the Tanner graph, are also important. Consequently, in order to achieve good decoding performance, we must ensure that we have an acceptable girth. Moreover, it is well known that there is a correspondence 
between short cycles in the Tanner graph and low-weight codewords for certain structured codes. In the following, we will consider $(2, K)$ - and $(3, K)$-regular QC-LDPC codes.

- For a $(2, K)$-regular code, $g=2 d_{\text {min }}$, since each codeword corresponds directly to a cycle or a union of edgedisjoint cycles [35].

- If $\mathbf{H}=\mathbf{B}^{\circlearrowleft N}$ is lifted from the $3 \times K$ all-ones base matrix $\mathbf{B}$, then we obtain the minimum distance bound $d_{\text {min }} \leq 24$ using Theorem 1 In this case, the existence of a 4 - or 6-cycle in the Tanner graph automatically implies a codeword of weight less than the upper bound $d_{\min } \leq$ 24 (see Theorems 22 and 25 in [21]). Consequently, a minimum girth of 8 is required to achieve the minimum distance bound.

It is well known that the girth of the Tanner graph associated with a parity-check matrix composed of circulant permutation matrices can be determined quickly using modular arithmetic [10], [26]. In [36], a technique was presented to derive a set of conditions on the permutation matrices of a protographbased parity-check matrix $\mathbf{H}=\mathbf{B}^{\uparrow N} \in \xi_{\mathbf{B}}(N)$ in order to achieve a certain desired girth $g$. It was shown that, if certain products of the permutation matrices comprising $\mathbf{H}$ do not have any fixed columns, then the girth will be at least $g$. In this paper, we construct protograph-based QC-LDPC codes with large minimum distance and use these conditions on the permutation matrices in order to achieve a certain guaranteed girth.

\section{PROTOGRAPH-BASED LDPC CODES OBTAINED BY "PRE-LIFTING" A PROTOGRAPH: DESIGN AND ANALYSIS}

In this section, we introduce a two-step lifting procedure based on a protograph. Based on this procedure, we describe how to construct QC-LDPC codes with increased girth and minimum distance while maintaining the circulant-based structure that facilitates efficient implementation. In the following, we mostly focus on base matrices $\mathbf{B}$ with only zero and one entries, i.e., protographs without parallel edges. This assumption simplifies analysis and ensures that the resulting codes are amenable to low-complexity implementation. (We demonstrate in Section IV-D that the technique can also be successfully applied to base matrices with parallel edges.)

\section{A. Constructing QC-LDPC codes by prelifting}

The construction technique can be defined in two steps:

1) first, a "pre-lifting" step where we take a carefully chosen $m$-fold graph cover of the protograph with base matrix $\mathbf{B}=\left[B_{i, j}\right]_{n_{c} \times n_{v}}$, where $m$ is typically small, to form a pre-lifted base matrix

$$
\mathbf{B}^{\uparrow m}=\left[\mathbf{B}_{i, j}\right],
$$

where $\mathbf{B}_{i, j}$ is an $m \times m$ permutation matrix if $B_{i, j}=1$, or the $m \times m$ all zero matrix if $B_{i, j}=0$,

2) following this, a second $r$-fold lifting step where we take an $r$-fold graph cover of the pre-lifted protograph associated with $\mathbf{B}^{\uparrow m}$, where $r$ is typically large. The permutations are chosen to be circulant, creating a QCLDPC code with parity-check matrix

$$
\mathbf{H}=\mathbf{B}^{\uparrow m \circlearrowleft r}=\left[\mathbf{H}_{i, j}\right],
$$

where

$$
\mathbf{H}_{i, j}=\left(\mathbf{B}_{i, j}\right)^{\circlearrowleft r}
$$

is an $m r \times m r$ circulant-block permutation matrix (see Section (I).

The codes that are constructed using this technique are QC with period $m n_{v}$, i.e., cyclically shifting the $r$ symbols in each of the $m n_{v}$ blocks in a codeword by one position results in another codeword.

\section{B. Examples of pre-lifting}

Example 3. Consider the $(2,3)$-regular base matrix

$$
\mathbf{B}=\left[\begin{array}{lll}
1 & 1 & 1 \\
1 & 1 & 1
\end{array}\right]
$$

- (One-step circulant lifting) Any QC-LDPC code derived from B using a one-step circulant-based lifting, i.e., with parity-check matrix

$$
\mathbf{H}=\mathbf{B}^{\circlearrowleft N}=\left[\begin{array}{ccc}
\mathbf{I}_{a}^{N} & \mathbf{I}_{b}^{N} & \mathbf{I}_{c}^{N} \\
\mathbf{I}_{d}^{N} & \mathbf{I}_{e}^{N} & \mathbf{I}_{f}^{N}
\end{array}\right] \in \xi_{\mathbf{B}}^{Q C}(N) \subseteq \xi_{\mathbf{B}}(N),
$$

has its minimum distance upper bounded by $\left(n_{c}+1\right) !=6$ and its girth upper bounded by 12 . (Recall that, for a parity-check matrix with column weight $2, g=2 d_{\text {min }}$, since each codeword corresponds directly to a cycle or a union of edge-disjoint cycles.)

- (Pre-lifting) A pre-lifted QC-LDPC code is obtained from B using

- a pre-lifted base matrix of the form

$$
\mathbf{B}^{\uparrow m}=\left[\begin{array}{lll}
\mathbf{B}_{1,1} & \mathbf{B}_{1,2} & \mathbf{B}_{1,3} \\
\mathbf{B}_{2,1} & \mathbf{B}_{2,2} & \mathbf{B}_{2,3}
\end{array}\right] \in \xi_{\mathbf{B}}(m),
$$

where each $\mathbf{B}_{i, j}$ is an $m \times m$ permutation matrix. - an $r$-lifting of $\mathbf{B}^{\uparrow m}$ to $\mathbf{B}^{\uparrow m \circlearrowleft r}$ to obtain

$$
\begin{aligned}
\mathbf{H} & =\mathbf{B}^{\uparrow m \circlearrowleft r}=\left[\begin{array}{lll}
\mathbf{H}_{1,1} & \mathbf{H}_{1,2} & \mathbf{H}_{1,3} \\
\mathbf{H}_{2,1} & \mathbf{H}_{2,2} & \mathbf{H}_{2,3}
\end{array}\right] \\
& \in \xi_{\mathbf{B}^{\uparrow C}}^{Q C}(r) \subseteq \xi_{\mathbf{B}}(m r),
\end{aligned}
$$

where each $\mathbf{H}_{i, j}$ is obtained by replacing every one in $\mathbf{B}_{i, j}$ with an $r \times r$ circulant permutation matrix.

- (Numerical pre-lifting example) Consider the following pre-lifted base matrix with $m=2$ :

$$
\begin{aligned}
\mathbf{B}^{\uparrow 2} & =\left[\begin{array}{lll}
\mathbf{B}_{1,1} & \mathbf{B}_{1,2} & \mathbf{B}_{1,3} \\
\mathbf{B}_{2,1} & \mathbf{B}_{2,2} & \mathbf{B}_{2,3}
\end{array}\right] \\
& =\left[\begin{array}{ll|ll|ll}
1 & 0 & 1 & 0 & 1 & 0 \\
0 & 1 & 0 & 1 & 0 & 1 \\
\hline 1 & 0 & 1 & 0 & 0 & 1 \\
0 & 1 & 0 & 1 & 1 & 0
\end{array}\right] \in \xi_{\mathbf{B}}(2) .
\end{aligned}
$$

Any code drawn from the QC-LDPC code ensemble based on this pre-lifted base matrix $\mathbf{B}^{\uparrow 2}$ has its minimum distance and girth bounded above by 10 and 20, respectively, which exceeds the upper bounds associated with 
the original base matrix $\mathbf{B}$. The following circulant-based lifting of $\mathbf{B}^{\uparrow 2}$ with $r=20$,

$$
\begin{aligned}
\mathbf{H} & =\mathbf{B}^{\uparrow 2 \circlearrowleft 20}=\left[\begin{array}{ccc|cc}
\mathbf{H}_{1,1} & \mathbf{H}_{1,2} & \mathbf{H}_{1,3} \\
\mathbf{H}_{2,1} & \mathbf{H}_{2,2} & \mathbf{H}_{2,3}
\end{array}\right] \\
& =\left[\begin{array}{cc|cccc}
\mathbf{I}_{0}^{20} & \mathbf{0} & \mathbf{I}_{0}^{20} & \mathbf{0} & \mathbf{I}_{0}^{20} & \mathbf{0} \\
\mathbf{0} & \mathbf{I}_{0}^{20} & \mathbf{0} & \mathbf{I}_{0}^{20} & \mathbf{0} & \mathbf{I}_{0}^{20} \\
\hline \mathbf{I}_{0}^{20} & \mathbf{0} & \mathbf{I}_{1}^{20} & \mathbf{0} & \mathbf{0} & \mathbf{I}_{0}^{20} \\
\mathbf{0} & \mathbf{I}_{0}^{20} & \mathbf{0} & \mathbf{I}_{9}^{20} & \mathbf{I}_{4}^{20} & \mathbf{0}
\end{array}\right] \\
& \in \xi_{\mathbf{B}^{Q C}}^{Q C}(20) \subseteq \xi_{\mathbf{B}}(40),
\end{aligned}
$$

defines a $[120,41,10]$ QC code with girth $g=20$, i.e., it achieves the improved upper bounds 3 Note that

$$
\mathbf{H}=\left[\begin{array}{ccc}
\mathbf{I}_{0}^{40} & \mathbf{I}_{0}^{40} & \mathbf{I}_{0}^{40} \\
\mathbf{I}_{0}^{40} & \mathbf{P} & \mathbf{Q}
\end{array}\right]
$$

but the permutation matrices $\mathbf{P}$ and $\mathbf{Q}$ are not circulant, i.e., they cannot be written in the form $\mathbf{I}_{a}^{40}$ for some integer $a$. Thus the bound $d_{\min } \leq 6$ does not apply.

Remark 3 Clearly, the pre-lifted base matrix $\mathbf{B}^{\uparrow m}$ defines a code that exists in the ensemble of all codes lifted from $\mathbf{B}$ with lifting factor $m, \xi_{\mathbf{B}}(m)$, and the QC code with paritycheck matrix $\mathbf{H}=\mathbf{B}^{\uparrow m \circlearrowleft r}$ obtained after the circulant lifting step exists in $\xi_{\mathbf{B}}(m r)$; however, $\mathbf{H}$ does not necessarily exist in $\xi_{\mathbf{B}}^{Q C}(m r)$, and thus the minimum distance may exceed $\left(n_{c}+1\right)$ !. Note that, since $\mathbf{H} \in \xi_{\mathbf{B}}(m r)$, the resulting code preserves the local graph neigbourhood structure and degree distribution of the protograph. Moreover, because $\mathbf{H}$ is composed of circulants, we maintain the efficient implementation advantages of QC codes.

Our goal in this paper is to study this two-step lifting process and determine how to construct QC-LDPC codes based on protographs with improved minimum distance and a certain guaranteed girth compared to one-step circulant-based liftings. In the following, we investigate the effect of pre-lifting a protograph on girth and minimum distance.

\section{Girth properties of pre-lifted protographs}

In this section, we will establish some results on the girth of a parity-check matrix obtained from a pre-lifted base matrix $\mathbf{B}^{\uparrow m}$. These results will later be used to obtain pre-lifted QCLDPC codes with a certain desired girth. This is important because short cycles have an adverse effect when decoding LDPC codes using iterative BP decoding. Also, there is a close connection between short cycles and low-weight codewords. In this regard, the structure imposed by a protograph is important.

It is well known that any cycle in a graph cover can be mapped to a cycle in the base graph (or protograph). As a direct consequence, we state the following result.

Lemma 4 If a protograph has girth $g$, then the girth of any $N$-lifted graph is bounded below by $g$.

\footnotetext{
${ }^{3}$ The parity-check matrix $\mathbf{H}$ has rank 79 , and hence the dimension of the code is $k=41$.
}

Theorem 4 implies the following corollary concerning the girth of a pre-lifted base matrix.

Corollary 5 If a pre-lifted base matrix $\mathbf{B}^{\uparrow m}$ has girth $g$, then the girth of any code from the ensemble $\xi_{\mathbf{B}^{\uparrow m}}^{Q C}(r)$ is bounded below by $g$, for any lifting factor $r$.

Design implications of Corollary 5 . One could use a technique such as progressive edge growth (PEG) [37] to design a pre-lifted base graph with girth $g$ and then, by using circulant permutations at the second lifting step, construct a QC-LDPC code with girth at least as large as $g$. However, even if the permutation matrices $\mathbf{B}_{i, j}$ chosen in the first lifting step do not satisfy the conditions needed to guarantee girth $g$, these conditions can still be satisfied in the second lifting step by carefully choosing the circulant matrices $\mathbf{I}_{s_{i, j, k}}^{r}$ comprising $\mathbf{H}_{i, j}$. Moreover, obtaining girth $g$ in the first lifting step will typically require a large lifting factor $m$, where we want $m$ to be as small as possible in order to simplify the analysis and implementation. An example is given in Section $\mathrm{V}$-A

If we wish to increase the girth from that of the pre-lifted base matrix $\mathbf{B}^{\uparrow m}$, it is necessary to check if certain products of the circulant-block permutation matrices comprising $\mathbf{H}=$ $\mathbf{B}^{\uparrow m \circlearrowleft r}$ have fixed columns (see Section IV-B). The following lemma proves useful to reduce the number of such conditions that one needs to check.

Lemma 6 Let $\mathbf{P}$ and $\mathbf{Q}$ be two $m r \times m r$ circulant-block permutation matrices derived from $m \times m$ permutation matrices $\mathbf{B}_{P}$ and $\mathbf{B}_{Q}$, respectively. Then the product $\mathbf{P Q}$ cannot have a fixed column if $\mathbf{B}_{P} \mathbf{B}_{Q}$ does not have a fixed column.

Proof. See Appendix B.

\section{Minimum distance properties of pre-lifted protograph- based codes}

In [25], MacKay and Davey established that, for a paritycheck matrix with an $n_{c} \times n_{v}$ grid of commuting permutation matrices, the minimum distance is bounded above by $\left(n_{c}+1\right)$ ! (cf. Theorem 1). We now establish a similar result for a grid of commuting circulant-block permutation matrices based on a pre-lifted base matrix $\mathbf{B}^{\uparrow m}$. To prove this, we require the following Lemma.

Lemma 7 Suppose that two circulant-block permutation matrices are given as

$$
\begin{aligned}
& \mathbf{P}=\operatorname{diag}\left(\mathbf{I}_{p_{1}}^{r}, \mathbf{I}_{p_{1}}^{r}, \ldots, \mathbf{I}_{p_{1}}^{r}\right) \cdot \tilde{\mathbf{B}}_{P}=\mathbf{B}_{P} \otimes \mathbf{I}_{p_{1}}^{r}, \\
& \mathbf{Q}=\operatorname{diag}\left(\mathbf{I}_{q_{1}}^{r}, \mathbf{I}_{q_{1}}^{r}, \ldots, \mathbf{I}_{q_{1}}^{r}\right) \cdot \tilde{\mathbf{B}}_{Q}=\mathbf{B}_{Q} \otimes \mathbf{I}_{q_{1}}^{r},
\end{aligned}
$$

where $p_{1}, q_{1} \in[r]$ and $\mathbf{B}_{P}$ and $\mathbf{B}_{Q}$ are $m \times m$ permutation matrices. Then,

$$
\mathbf{P Q}=\mathbf{Q P} \quad \text { iff } \quad \mathbf{B}_{P} \mathbf{B}_{Q}=\mathbf{B}_{Q} \mathbf{B}_{P} .
$$

Proof. See Appendix C. 
Then, the main result follows.

Theorem $\mathbf{8}$ Let $\mathbf{B}^{\uparrow m}$ be a pre-lifted base matrix derived from an $n_{c} \times n_{v}$ binary base matrix $\mathbf{B}$, and suppose

$$
\mathbf{B}_{i, j} \mathbf{B}_{k, l}=\mathbf{B}_{k, l} \mathbf{B}_{i, j},
$$

for all $i, k \in\left\{1,2, \ldots, n_{c}\right\}, j, l \in\left\{1,2, \ldots, n_{v}\right\},(i, j) \neq$ $(k, l)$. If

$$
s_{i, j, 1}=s_{i, j, 2}=\cdots=s_{i, j, m},
$$

for each circulant-block permutation matrix $\mathbf{H}_{i, j}$, as defined in (8), then the minimum distance of any code $C \in \xi_{\mathbf{B} \uparrow m}^{Q C}(r)$ is bounded above by $\left(n_{c}+1\right)$ !.

Proof. By applying Lemma 7 to each pair of circulant-block permutation matrices $\left(\mathbf{H}_{i, j}, \mathbf{H}_{k, l}\right), \forall i, k \in\left\{1,2, \ldots, n_{c}\right\}$, $j, l \in\left\{1,2, \ldots, n_{v}\right\}$, corresponding to the pair $\left(\mathbf{B}_{i, j} \mathbf{B}_{k, l}\right)$, we find that all pairs of matrices commute and thus the result of Theorem 1 holds.

Design Implications of Theorem 8 . In order to have minimum distance exceeding $\left(n_{c}+1\right)$ !, we must have at least one pair of non-commuting circulant-block permutation matrices. In fact, we require that at least one pair of circulant-block matrices is strongly noncommutative. Note that, in general, if

$$
\begin{aligned}
& \mathbf{P}=\operatorname{diag}\left(\mathbf{I}_{p_{1}}^{r}, \mathbf{I}_{p_{2}}^{r}, \ldots, \mathbf{I}_{p_{m}}^{r}\right) \cdot \tilde{\mathbf{B}}_{P}, \\
& \mathbf{Q}=\operatorname{diag}\left(\mathbf{I}_{q_{1}}^{r}, \mathbf{I}_{q_{2}}^{r}, \ldots, \mathbf{I}_{q_{m}}^{r}\right) \cdot \tilde{\mathbf{B}}_{Q},
\end{aligned}
$$

then, as described in the proof of Lemma 6

$$
\begin{aligned}
& \mathbf{P Q}=\operatorname{diag}\left(\mathbf{I}_{p_{1}+q_{\sigma(1)}}^{r}, \mathbf{I}_{p_{2}+q_{\sigma(2)}}^{r}, \ldots, \mathbf{I}_{p_{m}+q_{\sigma(m)}}^{r}\right) \cdot \tilde{\mathbf{B}}_{P} \tilde{\mathbf{B}}_{Q}, \\
& \mathbf{Q P}=\operatorname{diag}\left(\mathbf{I}_{q_{1}+p_{\tau(1)}}^{r}, \mathbf{I}_{q_{2}+p_{\tau(2)}}^{r}, \ldots, \mathbf{I}_{q_{m}+p_{\tau(m)}}^{r}\right) \cdot \tilde{\mathbf{B}}_{Q} \tilde{\mathbf{B}}_{P},
\end{aligned}
$$

where $\sigma$ and $\tau$ are the permutations associated with $\mathbf{B}_{P}$ and $\mathbf{B}_{Q}$, respectively, and addition is performed modulo $r$. In addition,

$$
\tilde{\mathbf{B}}_{P} \tilde{\mathbf{B}}_{Q}=\left(\mathbf{B}_{P} \otimes \mathbf{I}_{0}^{r}\right)\left(\mathbf{B}_{Q} \otimes \mathbf{I}_{0}^{r}\right)=\mathbf{B}_{P} \mathbf{B}_{Q} \otimes \mathbf{I}_{0}^{r} \mathbf{I}_{0}^{r}
$$

by the distributive law of the Kronecker product, and it follows that

$$
\tilde{\mathbf{B}}_{P} \tilde{\mathbf{B}}_{Q}=\tilde{\mathbf{B}}_{Q} \tilde{\mathbf{B}}_{P} \Longleftrightarrow \mathbf{B}_{P} \mathbf{B}_{Q}=\mathbf{B}_{Q} \mathbf{B}_{P} .
$$

Consequently, to ensure $\mathbf{P}$ and $\mathbf{Q}$ are strongly noncommutative, we consider two cases:

- if $\mathbf{B}_{P} \mathbf{B}_{Q}=\mathbf{B}_{Q} \mathbf{B}_{P}$, then we see from (10), (11), and (12) that we must ensure that the diagonal matrices in (10) and (11) do not have an overlapping column;

- if $\mathbf{B}_{P}$ and $\mathbf{B}_{Q}$ are strongly noncommutative, then $\mathbf{P}$ and $\mathbf{Q}$ are also strongly noncommutative, even if the diagonal matrices in (10) and (11) are equal.

In the next section, we will use these two cases to propose two new design rules for constructing QC-LDPC codes based on a pre-lifted protograph.

\section{E. Designing good pre-lifted protographs}

In order to avoid being constrained by the upper bound of Theorem 8 , it is necessary to ensure that there is at least one pair of strongly noncommutative circulant-block permutation matrices in $\mathbf{H}$ (see the discussion of Theorem 8 in Section $\amalg I-D$. We now provide two new design rules for constructing QC-LDPC codes based on a pre-lifted protograph depending on whether the permutation matrices used for prelifting commute or not.

- Design Rule 1: Commuting pre-lifting permutation matrices. In this case, at Step 1, each pair of matrices $\mathbf{B}_{i, j}$ and $\mathbf{B}_{k, l},(i, j) \neq(k, l)$, is chosen to be commuting. (Typically, we choose circulant matrices in applying Design Rule 1 , since they necessarily commute.) At the second step, since the pre-lifting permutation matrices commute, we must ensure that the diagonal matrices are chosen such that at least one pair of circulant-block matrices $(\mathbf{P}, \mathbf{Q})$ in $\mathbf{H}$ is strongly noncommutative, i.e.,

$$
p_{i}+q_{\sigma(i)} \not \equiv q_{i}+p_{\tau(i)} \quad \bmod r,
$$

for all $i \in\{1,2, \ldots, m\}$ (thus necessarily $\mathbf{P Q} \neq \mathbf{Q P}$ ). This can be achieved, for example, by imposing the condition that $\tau$ has no fixed point, setting $q_{1}=q_{2}=$ $\cdots=q_{m}$, and choosing each $p_{i}$ to be distinct.

- Design Rule 2: Non-commuting pre-lifting permutation matrices. In Step 1, we choose permutation matrices $\mathbf{B}_{i, j}$ and ensure that at least one pair of matrices $\left(\mathbf{B}_{i, j}, \mathbf{B}_{k, l}\right)$, $(i, j) \neq(k, l)$, is strongly noncommutative, thus necessarily

$$
\mathbf{B}_{i, j} \mathbf{B}_{k, l} \neq \mathbf{B}_{k, l} \mathbf{B}_{i, j} .
$$

At Step 2, we then choose all circulant permutation matrices in each circulant-block matrix to have the same shift parameter, i.e., $p_{1}=p_{2}=\cdots=p_{m}$ for circulantblock $\mathbf{P}$.

These design rules give necessary (but not sufficient) conditions for pre-lifted QC-LDPC codes to have minimum distance exceeding that of QC-LDPC codes lifted directly from B. Note that the rules above apply directly when $n_{v}=n_{c}+1$; however, when $n_{v}>n_{c}+1$, they must be applied to every $n_{c} \times\left(n_{c}+1\right)$ block submatrix.

We will later give examples of how the permutations at both steps should be chosen to ensure large minimum distance and girth. In Section IV we focus on pre-lifting (Step 1) and discuss choosing permutations to maximize the distance upper bound calculated from (6). At the pre-lifting step, the conditions on the circulants that must be checked to guarantee a desired girth $g$ at the next step can be determined, and we demonstrate in Section $\nabla$ that certain choices of prelifting can reduce the number of conditions to be checked or even eliminate the need to check any conditions. For both design rules, we then provide examples in Sections IV] VI] of circulants chosen at Step 2 that result in improved minimum distance and achieve a desired girth $g$.

Remark 9 By ensuring that some pairs of circulant-block permutation matrices $\mathbf{H}_{i, j}$ are strongly noncommutative, we can construct pre-lifted QC-LDPC codes with minimum distance 
exceeding $\left(n_{c}+1\right)$ !. This can be observed by examining the proof of Theorem 2 in [25]. When some pairs of permutation matrices are strongly noncommutative, instead of finding a codeword of weight $\left(n_{c}+1\right)$ !, we obtain a binary vector of weight $\left(n_{c}+1\right)$ ! that has a small number $f>0$ of unsatisfied parity-check equations (commonly referred to as a $\left(\left(n_{c}+1\right) !, f\right)$ near-codeword $)$. An example is given in Appendix D

Remark 10 In general, when constructing short to moderate length parity-check matrices $\mathbf{H}=\left[\mathbf{H}_{i, j}\right]$, it is a difficult problem to search for permutation matrices $\mathbf{H}_{i, j}$ such that the code achieves a desired minimum distance and girth. We will see in the following sections that this search is much simpler if we construct the parity-check matrices using a twostep method and circulant-block permutation matrices $\mathbf{H}_{i, j}$.

\section{Code Design: Selecting PERMUTATIONS FOR PRE-LIFTING}

In this section, we focus on the selection process for the permutations involved in the first step of the construction technique by considering two examples: a simple $(2,3)$-regular protograph that is useful in describing the method and is easy to analyze, and a more practically interesting $(3,4)$-regular protograph that demonstrates the successful application of the method to a protograph with larger node degrees.

- For the first example, we show that the upper bounds on minimum distance and girth obtained for the original base matrix can be increased by pre-lifting the protograph and that the new upper bounds increase as larger degrees of pre-lifting are considered. We demonstrate that the improved minimum distance and girth promised by the increased upper bounds are indeed obtainable by selecting appropriate circulants at the second lifting step and we give explicit constructions showing the increased bounds are in fact tight.

- For the second example, we show that even larger gains in minimum distance are possible. In particular, we show that the upper bound on minimum distance can be increased significantly by pre-lifting and confirm the improvement by providing specific constructions with improved minimum distance (larger than the original upper bounds) and a certain guaranteed girth.

\section{A. Pre-lifted $Q C$ structures for a $2 \times 3$ base matrix}

We begin our study with a base matrix of column weight 2 ; in particular, the $(2,3)$-regular base matrix $\mathbf{B}$ discussed in Example 3

- Any $N$-fold graph cover of B can be written in the form of (2), i.e.,

$$
\mathbf{H}=\mathbf{B}^{\uparrow N}=\left[\begin{array}{ccc}
\mathbf{I}_{0}^{N} & \mathbf{I}_{0}^{N} & \mathbf{I}_{0}^{N} \\
\mathbf{I}_{0}^{N} & \mathbf{P} & \mathbf{Q}
\end{array}\right] \in \xi_{\mathbf{B}}(N),
$$

where $\mathbf{P}$ and $\mathbf{Q}$ are two permutation matrices of size $N \times N$.

- Recall that, by applying Theorem 2 to the base matrix $\mathbf{B}$, we find that any code drawn from the QC sub-ensemble
$\xi_{\mathrm{B}}^{Q C}(N)$ has minimum distance at most 6 , or equivalently, girth at most 12 . In other words, we cannot exceed a girth of 12 unless we choose non-circulant permutation matrices $\mathbf{P}$ and $\mathbf{Q}$.

1) Pre-lifting a $2 \times 3$ protograph:

- A pre-lifted base matrix $\mathbf{B}^{\uparrow m}$ can be written, without loss of generality, as

$$
\mathbf{B}^{\uparrow m}=\left[\begin{array}{ccc}
\mathbf{I}_{0}^{m} & \mathbf{I}_{0}^{m} & \mathbf{I}_{0}^{m} \\
\mathbf{I}_{0}^{m} & \mathbf{B}_{2,2} & \mathbf{B}_{2,3}
\end{array}\right] \in \xi_{\mathbf{B}}(m) .
$$

Note that, since $\mathbf{B}^{\uparrow m}$ is $m$-lifted from $\mathbf{B}$, the search space for good pre-lifted base matrices $\mathbf{B}^{\uparrow m}$ consists of at most $m !^{2}$ combinations of permutation matrices, where $m$ is typically a small integer.

- A QC-LDPC code can now be $r$-lifted from $\mathbf{B}^{\uparrow m}$ as

$$
\begin{aligned}
\mathbf{H} & =\mathbf{B}^{\uparrow m \circlearrowleft r} \\
& =\left[\begin{array}{ccc}
\left(\mathbf{I}_{0}^{m}\right)^{\circlearrowleft r} & \left(\mathbf{I}_{0}^{m}\right)^{\circlearrowleft r} & \left(\mathbf{I}_{0}^{m}\right)^{\circlearrowleft r} \\
\left(\mathbf{I}_{0}^{m}\right)^{\circlearrowleft r} & \left(\mathbf{B}_{2,2}\right)^{\circlearrowleft r} & \left(\mathbf{B}_{2,3}\right)^{\circlearrowleft r}
\end{array}\right] \in \xi_{\mathbf{B} \uparrow m}^{Q C}(r) .
\end{aligned}
$$

- Continuing, it can easily be shown (see Appendix A that by row and column permutations any parity-check matrix $\mathbf{H} \in \xi_{\mathbf{B} \uparrow m}^{Q C}(r)$ can be re-written as

$$
\begin{aligned}
\mathbf{H} & =\mathbf{B}^{\uparrow m \circlearrowleft r} \\
& =\left[\begin{array}{ccc}
\mathbf{I}_{0}^{m r} & \mathbf{I}_{0}^{m r} & \mathbf{I}_{0}^{m r} \\
\mathbf{I}_{0}^{m r} & \left(\mathbf{B}_{2,2}\right)^{\circlearrowleft r} & \left(\mathbf{B}_{2,3}\right)^{\circlearrowleft r}
\end{array}\right] \in \xi_{\mathbf{B}^{\uparrow C}}^{Q C}(r) .
\end{aligned}
$$

(Note that, as a result of the row and column permutations, the matrices $\mathbf{B}_{2,2}$ and $\mathbf{B}_{2,3}$ in 15 are different than the corresponding matrices in (14).) Similar to the simplified representation of $\mathbf{B}^{\uparrow m}$ using identity matrices in (14), the motivation to write $\mathbf{H}$ in the form (15) is to simplify the search for suitable circulant permutation matrices at the second lifting step. Instead of searching through $\mathrm{m}^{6}$ combinations of circulants, the search space for good QC-LDPC codes is thus reduced to $\mathrm{m}^{2}$ combinations of circulants.

For example, consider the pre-lifted base matrix $\mathbf{B}^{\uparrow 2}$ chosen in (9). Every parity-check matrix $\mathbf{H}=\mathbf{B}^{\uparrow 2 \circlearrowleft r}$ in the ensemble $\xi_{\mathbf{B} \uparrow 2}^{Q C}(r)$ can be written in the form

$$
\begin{aligned}
\mathbf{H} & =\mathbf{B}^{\uparrow 2 \circlearrowleft r}=\left[\begin{array}{cc|cc|cc}
\mathbf{I}_{0}^{r} & \mathbf{0} & \mathbf{I}_{0}^{r} & \mathbf{0} & \mathbf{I}_{0}^{r} & \mathbf{0} \\
\mathbf{0} & \mathbf{I}_{0}^{r} & \mathbf{0} & \mathbf{I}_{0}^{r} & \mathbf{0} & \mathbf{I}_{0}^{r} \\
\hline \mathbf{I}_{0}^{r} & \mathbf{0} & \mathbf{I}_{p_{1}}^{r} & \mathbf{0} & \mathbf{0} & \mathbf{I}_{q_{1}}^{r} \\
\mathbf{0} & \mathbf{I}_{0}^{r} & \mathbf{0} & \mathbf{I}_{p_{2}}^{r} & \mathbf{I}_{q_{2}}^{r} & \mathbf{0}
\end{array}\right] \\
& =\left[\begin{array}{ccc}
\mathbf{I}_{0}^{2 r} & \mathbf{I}_{0}^{2 r} & \mathbf{I}_{0}^{2 r} \\
\mathbf{I}_{0}^{2 r} & \mathbf{P} & \mathbf{Q}
\end{array}\right]
\end{aligned}
$$

for some $p_{1}, p_{2}, q_{1}, q_{2} \in[r]$.

- By applying Theorem 2 , we find that a code $\mathcal{C}$ drawn from the QC sub-ensemble $\xi_{\mathbf{B}^{\uparrow 2}}^{Q C}(r)$ with base matrix $\mathbf{B}^{\uparrow 2}$ from (9) has its minimum distance bounded above by $d_{\min } \leq$ 10 (and hence its girth bounded above by $g \leq 20$ ).

- Note that, by choosing $m=2$, we are forced to use Design Rule 1, because permutations of size 2 automatically commute. As such, if we hope to achieve increased minimum distance, we must ensure $p_{1}, p_{2}, q_{1}$, and $q_{2}$ are chosen such that $\mathbf{P Q}$ and QP do not have an overlapping column. 
It can easily be shown that the improvement in minimum distance and girth promised by the application of Theorem 2 can be achieved by codes from $\xi_{\mathbf{B} \uparrow m}^{Q C}(r)$. For example,

- choosing lifting factor $r=9$ and $\left(p_{1}, p_{2}, q_{1}, q_{2}\right)=$ $(1,2,0,6)$, gives a $[54,19,8]$ code with girth $g=16$;

- choosing lifting factor $r=20$ and $\left(p_{1}, p_{2}, q_{1}, q_{2}\right)=$ $(1,9,0,4)$, gives a $[120,41,10]$ code with girth $g=20$.

For the pre-lifting configuration of (16), we find that $r=9$ and $r=20$ are the smallest possible circulant sizes that enable us to construct codes with girths 16 and 20, corresponding to minimum distances $d_{\min }=8$ and $d_{\min }=10$, respectively. There are 216 (resp. 2880) such codes in the $r=9$ (resp. $r=20$ ) QC sub-ensembles.

Remark 11 By choosing $\mathbf{P}$ and $\mathbf{Q}$ to be arrays of circulants, or circulant-block matrices, rather than just searching for random permutations, we obtain a significant improvement in both the girth and the minimum distance compared to a direct circulant lifting while maintaining the desirable circulant structure facilitating simplified encoding and decoding. Moreover, the search space for good codes is greatly reduced. In searching for a code with pre-lifting factor $m$ and circulant lifting factor $r$, the circulant-block permutation matrix $\mathbf{P}$ has $m ! \cdot r^{m}$ choices, or $r^{m}$ choices after the pre-lifting stage, whereas there are $(m r)$ ! choices for a general permutation matrix $\mathbf{P}$ of size $m r$. For example, in searching for a code with minimum distance $d_{\min }=8$ when $m=2$ and $r=9$, there are $m ! \cdot r^{m}=162$ choices before pre-lifting, or $r^{m}=81$ choices after pre-lifting, for each of the circulant-block permutation matrices, whereas there are $m r !=18 ! \approx 6.4 \times 10^{15}$ choices for a random permutation matrix of size $m r=18$. Note that the number of choices grows quickly with $m$; thus the prelifting factor $m$ should be chosen to be small.

2) Choosing $m$-fold graph covers for pre-lifting a protograph: Not all choices of covering graphs are equivalent at the pre-lifting step. For example, the possible choices for the submatrix $\left[\mathbf{B}_{2,2} \mid \mathbf{B}_{2,3}\right]$ in (14) at the pre-lifting step are

$$
\begin{aligned}
& {\left[\begin{array}{ll|ll}
1 & 0 & 1 & 0 \\
0 & 1 & 0 & 1
\end{array}\right],} \\
& {\left[\begin{array}{ll|ll}
1 & 0 & 0 & 1 \\
0 & 1 & 1 & 0
\end{array}\right],} \\
& {\left[\begin{array}{ll|ll}
0 & 1 & 1 & 0 \\
1 & 0 & 0 & 1
\end{array}\right],} \\
& {\left[\begin{array}{ll|ll}
0 & 1 & 0 & 1 \\
1 & 0 & 1 & 0
\end{array}\right] .}
\end{aligned}
$$

Note that choices (18), (19), and (20) result in equivalent base matrices $\mathbf{B}^{\uparrow 2}$, i.e., they can be shown to be equal using only elementary row and column operations. Consequently, their lifted ensembles $\xi_{\mathbf{B} \uparrow 2}^{Q C}(r)$ consist of the same set of codes, up to row and column permutations.

Applying the bound (6) to the pre-lifted configuration (17), we find that a code $\mathcal{C}$ from the $\mathrm{QC}$ sub-ensemble $\xi_{\mathbf{B} \uparrow 2}^{Q C}(r)$ has its minimum distance bounded above by $d_{\min } \leq 12$. However, note that the Tanner graph of base matrix $\mathbf{B}^{\uparrow 2}$ corresponding to (17) consists of two disconnected copies of the original protograph. It follows that any lifted parity-check matrix contains the two following disjoint substructures:

$$
\left[\begin{array}{ccc}
\mathbf{I}_{0}^{r} & \mathbf{I}_{0}^{r} & \mathbf{I}_{0}^{r} \\
\mathbf{I}_{0}^{r} & \mathbf{I}_{p_{1}}^{r} & \mathbf{I}_{q_{1}}^{r}
\end{array}\right] \text { and }\left[\begin{array}{ccc}
\mathbf{I}_{0}^{r} & \mathbf{I}_{0}^{r} & \mathbf{I}_{0}^{r} \\
\mathbf{I}_{0}^{r} & \mathbf{I}_{p_{2}}^{r} & \mathbf{I}_{q_{2}}^{r}
\end{array}\right]
$$

and consequently its minimum distance and girth are bounded above by $d_{\min } \leq 6$ and $g \leq 12$, respectively. Thus, in terms of maximizing minimum distance and girth, the pre-lifting configuration (18), or the equivalent pre-lifting configurations (19) or 20), should be chosen.

3) Larger degrees of pre-lifting: Intuitively, the larger we make the pre-lifting factor $m$ for a fixed block length $n$, the more 'random-like' the QC sub-ensemble $\xi_{\mathrm{B} \uparrow m}^{Q C}(r)$ becomes and, as a consequence, we would expect the maximum achievable minimum distance to increase. We have seen that, for $m=2$, the maximum achievable minimum distance of a circulant-based lifting increased from $d_{\min } \leq 6$ to $d_{\min } \leq 10$, and correspondingly, the maximum achievable girth increased from 12 to 20 . In the remainder of this section, we describe how the minimum distance and girth are affected by increasing the pre-lifting factor to values of $m \geq 3$. Note that, for $m \geq 3$, the permutation matrices do not necessarily commute with one another, so both Design Rules 1 and 2 may be used.

We employ the sieve principle (start with all possibilities, perform a test, remove candidates that fail the test, and repeat until we can no longer separate the candidates) in order to find a good covering graph to use at the pre-lifting step. Note that every 3 -cover can be written in the form of (14), and as such, there are $m !^{2}=3 !^{2}=36$ covering graphs to consider for $m=3$. Of these 3 -covers, we find that many are equivalent. In fact, after removing (or sieving out) equivalent graphs, we are left with only five choices. Of these choices, if any contain disjoint sub-graphs of a smaller covering graph ( $m=1$ or $m=2$ in this case), then the minimum distance cannot exceed the corresponding bound calculated for the subgraph. For a 3-cover, there are two such sub-graphs; either there are three copies of the 1-cover ( 3 disjoint copies of the original protograph), or the lifted graph consists of both a 1cover and a 2-cover (a copy of the original protograph and a disjoint 2-cover). In both cases, a code $\mathcal{C}$ drawn from the QC sub-ensemble has its minimum distance bounded above by $d_{\min } \leq 6$ as a result of the substructure associated with the 1-cover. For example, the only configuration of $\left[\mathbf{B}_{2,2} \mid \mathbf{B}_{2,3}\right]$ that results in three copies of the 1-cover is when both $\mathbf{B}_{2,2}$ and $\mathbf{B}_{2,3}$ are identity matrices, i.e., the circulants in the lifted circulant-block matrix occur only on the leading diagonal. There are nine (equivalent) occurrences of the second limiting substructure consisting of both a 1-cover and a 2-cover. One such example is the substructure

$$
\left[\mathbf{B}_{2,2} \mid \mathbf{B}_{2,3}\right]=\left[\begin{array}{ccc|ccc}
1 & 0 & 0 & 0 & 1 & 0 \\
0 & 1 & 0 & 1 & 0 & 0 \\
0 & 0 & 1 & 0 & 0 & 1
\end{array}\right]
$$

which again results in any code $\mathcal{C}$ drawn from $\xi_{\mathbf{B}^{\uparrow 3}}^{Q C}(r)$ having its minimum distance bounded above by $d_{\min } \leq 6$ for arbitrarily large circulant size $r$.

Note that applying (6) to base matrices containing these two harmful substructures gives the loose upper bounds $d_{\text {min }} \leq 24$ 
and $d_{\min } \leq 12$, respectively, and so it is necessary to remove these candidates before before proceeding with the code construction. After removing the equivalent covering graphs and those containing disjoint subgraphs, we are left with three candidates for the pre-lifted base matrix $\mathbf{B}^{\uparrow 3}$. Applying (6) to the remaining choices results in one candidate that bounds the minimum distance of circulant-based codes drawn from the ensemble by $d_{\min } \leq 10$ and two (non-equivalent) candidates with bound $d_{\min } \leq 12$. Note that $d_{\min } \leq 10$ is achievable by a 2 -cover, so this choice is removed, leaving only two remaining choices for the pre-lifted graph. One of the remaining choices is the 3-cover with the following sub-matrix (before and after the second lifting step)

$$
\begin{aligned}
{\left[\mathbf{B}_{2,2} \mid \mathbf{B}_{2,3}\right] } & =\left[\begin{array}{ccc|ccc}
1 & 0 & 0 & 0 & 1 & 0 \\
0 & 1 & 0 & 0 & 0 & 1 \\
0 & 0 & 1 & 1 & 0 & 0
\end{array}\right] \rightsquigarrow \\
{\left[\mathbf{H}_{2,2} \mid \mathbf{H}_{2,3}\right] } & =\left[\begin{array}{ccc|ccc}
\mathbf{I}_{p_{1}}^{r} & \mathbf{0} & \mathbf{0} & \mathbf{0} & \mathbf{I}_{q_{1}}^{r} & \mathbf{0} \\
\mathbf{0} & \mathbf{I}_{p_{2}}^{r} & \mathbf{0} & \mathbf{0} & \mathbf{0} & \mathbf{I}_{q_{2}}^{r} \\
\mathbf{0} & \mathbf{0} & \mathbf{I}_{p_{3}}^{r} & \mathbf{I}_{q_{3}}^{r} & \mathbf{0} & \mathbf{0}
\end{array}\right]
\end{aligned}
$$

Note that this choice of pre-lifting again forces us to use Design Rule 1, since the permutation matrices in $\mathbf{B}^{\uparrow 3}$ all commute with one another. Choosing circulants $\mathbf{I}_{p_{1}}^{46}, \mathbf{I}_{p_{2}}^{46}, \mathbf{I}_{p_{3}}^{46}, \mathbf{I}_{q_{1}}^{46}, \mathbf{I}_{q_{2}}^{46}$, and $\mathbf{I}_{q_{3}}^{46}$ as $\mathbf{I}_{1}^{46}, \mathbf{I}_{5}^{46}, \mathbf{I}_{25}^{46}, \mathbf{I}_{4}^{46}, \mathbf{I}_{7}^{46}$, and $\mathbf{I}_{28}^{46}$, respectively, results in a code $\mathcal{C}$ with minimum distance $d_{\text {min }}=12$ and girth $g=24$, and we see that the corresponding bound can be achieved.

The procedure can be repeated for $m \geq 4$. Applying the sieve technique to the $4 !^{2}$ candidate covering graphs for $m=4$, we are left with five candidates for which $d_{\min } \leq 14$. Codes achieving a minimum distance equal to 14 can be constructed, so we see again that the bound can be achieved. Table I summarizes the results we have obtained as a result of pre-lifting the $2 \times 3$ all-ones base matrix $\mathbf{B}$.

TABLE I

LARGEST ACHIEVABLE VALUES OF MINIMUM DISTANCE AND GIRTH FOR A $(2,3)$-REgUlAR BASE MATRIX GIVEN A PARTICULAR PRE-LIFTING FACTOR $m$.

\begin{tabular}{|c|c|c|}
\hline pre-lifting factor $m$ & $d_{\min }$ & girth \\
\hline 1 & 6 & 12 \\
2 & 10 & 20 \\
3 & 12 & 24 \\
4 & 14 & 28 \\
\hline
\end{tabular}

Note that the minimum distance grows slowly in this example, but this is expected for $(2,3)$-regular codes (see [4]). It does, however, demonstrate that the minimum distance and girth can be improved by pre-lifting the protograph. In the next section we will obtain larger improvements by considering a protograph with increased node degrees.

4) Discussion: The pre-lifting technique described above is a simple but effective way to improve the performance of QCLDPC codes. In fact, many existing QC-LDPC codes in the literature can be viewed as pre-lifted codes. In this subsection, we compare some of our constructions to known optimal and close to optimal codes (in the sense of minimal block length for a given $\left.d_{\min }\right)$. For example, we know that a code can be constructed with $d_{\min }=6$ by directly lifting $\mathbf{H}$ from $\mathbf{B}$, i.e., $m=1$. In fact, the minimal length $[21,8,6]$ code meeting this criteria can be viewed as a (degenerate) pre-lifted graph with $m=1$ and $r=7$, where the parity-check matrix

$$
\mathbf{H}=\left[\begin{array}{lll}
\mathbf{I}_{0}^{7} & \mathbf{I}_{0}^{7} & \mathbf{I}_{0}^{7} \\
\mathbf{I}_{0}^{7} & \mathbf{I}_{4}^{7} & \mathbf{I}_{6}^{7}
\end{array}\right]
$$

determines the Heawood graph [38]. To obtain $d_{\text {min }}=8$, we see from Table \ that it is necessary to increase the pre-lifting factor to at least $m=2$. It is known that the shortest possible (optimal) (2,3)-regular code has parameters [45, 16, 8] [38], which is not too far from the pre-lifted $[54,19,8]$ code constructed in Section IV-A-1 with $m=2$ and $r=9$ (which was obtained with no particular effort to minimize block length). In addition, we note that the optimal $[45,16,8]$ code [38] can be viewed as a pre-lifted code from $\mathbf{B}$ with $m=3$ and $r=5$, where the parity-check matrix is:

$$
\mathbf{H}=\left[\begin{array}{ccc|ccc|ccc}
\mathbf{I}_{0}^{5} & \mathbf{0} & \mathbf{0} & \mathbf{I}_{0}^{5} & \mathbf{0} & \mathbf{0} & \mathbf{I}_{0}^{5} & \mathbf{0} & \mathbf{0} \\
\mathbf{0} & \mathbf{I}_{0}^{5} & \mathbf{0} & \mathbf{0} & \mathbf{I}_{0}^{5} & \mathbf{0} & \mathbf{0} & \mathbf{I}_{0}^{5} & \mathbf{0} \\
\mathbf{0} & \mathbf{0} & \mathbf{I}_{0}^{5} & \mathbf{0} & \mathbf{0} & \mathbf{I}_{0}^{5} & \mathbf{0} & \mathbf{0} & \mathbf{I}_{0}^{5} \\
\hline \mathbf{I}_{0}^{5} & \mathbf{0} & \mathbf{0} & \mathbf{0} & \mathbf{0} & \mathbf{I}_{0}^{5} & \mathbf{0} & \mathbf{I}_{1}^{5} & \mathbf{0} \\
\mathbf{0} & \mathbf{I}_{0}^{5} & \mathbf{0} & \mathbf{I}_{0}^{5} & \mathbf{0} & \mathbf{0} & \mathbf{I}_{3}^{5} & \mathbf{0} & \mathbf{0} \\
\mathbf{0} & \mathbf{0} & \mathbf{I}_{0}^{5} & \mathbf{0} & \mathbf{I}_{4}^{5} & \mathbf{0} & \mathbf{0} & \mathbf{0} & \mathbf{I}_{1}^{5}
\end{array}\right] .
$$

Consequently, it is clear that, for a given desired block length $n=n_{v} m r$ and required $d_{\mathrm{min}}$, it is an interesting challenge to choose the correct degree of pre-lifting $m$. Generally, to reduce complexity, we choose $m$ as small as possible to achieve a desired $d_{\text {min }}$; however, as we see in this example, it is possible that such a $d_{\text {min }}$ can be obtained with a shorter overall block length by choosing a larger $m$ and smaller $r$.

Finally, we point out that for $d_{\min }=10$ the $[120,41,10]$ pre-lifted code constructed in Section IV-A-1 is also close to the optimal $[105,36,10]$ code based on the Balaban graph [38] and to the near-optimal $[108,37,10]$ code presented in [38]. The fact that the pre-lifted codes, which were constructed for demonstration purposes without any particular effort to minimize block length, are close to the lower bounds on block length for a given $d_{\text {min }}$ demonstrates the efficiency of this method. Useful references and short tables of near-optimal $(2, K)$-regular LDPC codes can be found in [38].

\section{B. Pre-lifted $Q C$ structures for a $3 \times 4$ base matrix}

Consider the $(3,4)$-regular protograph-based ensemble defined by the all-ones base matrix $\mathbf{B}$ of size $3 \times 4$.

- We can assume, without loss of generality, that any paritycheck matrix derived from $\mathbf{B}$ can be written in the form of (2), i.e.,

$$
\mathbf{H}=\mathbf{B}^{\uparrow N}=\left[\begin{array}{cccc}
\mathbf{I}_{0}^{N} & \mathbf{I}_{0}^{N} & \mathbf{I}_{0}^{N} & \mathbf{I}_{0}^{N} \\
\mathbf{I}_{0}^{N} & \mathbf{P} & \mathbf{R} & \mathbf{T} \\
\mathbf{I}_{0}^{N} & \mathbf{Q} & \mathbf{S} & \mathbf{U}
\end{array}\right] \in \xi_{\mathbf{B}}(N),
$$

where $\mathbf{P}, \mathbf{Q}, \mathbf{R}, \mathbf{S}, \mathbf{T}$, and $\mathbf{U}$ are $N \times N$ permutation matrices.

- We can also assume, without loss of generality, that a pre-lifted base matrix $\mathbf{B}^{\uparrow m}$ has the form

$$
\mathbf{B}^{\uparrow m}=\left[\begin{array}{cccc}
\mathbf{I}_{0}^{m} & \mathbf{I}_{0}^{m} & \mathbf{I}_{0}^{m} & \mathbf{I}_{0}^{m} \\
\mathbf{I}_{0}^{m} & \mathbf{B}_{2,2} & \mathbf{B}_{2,3} & \mathbf{B}_{2,4} \\
\mathbf{I}_{0}^{m} & \mathbf{B}_{3,2} & \mathbf{B}_{3,3} & \mathbf{B}_{3,4}
\end{array}\right] \in \xi_{\mathbf{B}}(m),
$$


where $\mathbf{B}_{2,2}, \mathbf{B}_{3,2}, \mathbf{B}_{2,3}, \mathbf{B}_{3,3}, \mathbf{B}_{2,4}$, and $\mathbf{B}_{3,4}$ are $m \times m$ permutation matrices.

- Finally, any parity-check matrix $\mathbf{H} \in \xi_{\mathbf{B}^{\uparrow m}}^{Q C}(r)$ can be written as

$$
\begin{aligned}
\mathbf{H} & =\mathbf{B}^{\uparrow m \circlearrowleft r} \\
& =\left[\begin{array}{cccc}
\mathbf{I}_{0}^{m r} & \mathbf{I}_{0}^{m r} & \mathbf{I}_{0}^{m r} & \mathbf{I}_{0}^{m r} \\
\mathbf{I}_{0}^{m r} & \left(\mathbf{B}_{2,2}\right)^{\circlearrowleft r} & \left(\mathbf{B}_{2,3}\right)^{\circlearrowleft r} & \left(\mathbf{B}_{2,4}\right)^{\circlearrowleft r} \\
\mathbf{I}_{0}^{m r} & \left(\mathbf{B}_{3,2}\right)^{\circlearrowleft r} & \left(\mathbf{B}_{3,3}\right)^{\circlearrowleft r} & \left(\mathbf{B}_{3,4}\right)^{\circlearrowleft r}
\end{array}\right]
\end{aligned}
$$

after row and column permutations. Note that (25) is in the form of (23), where $N=m r$ and $\mathbf{P}, \mathbf{Q}, \mathbf{R}, \mathbf{S}, \mathbf{T}$, and $\mathbf{U}$ are circulant-block permutation matrices. Note also that, as a result of the row and column permutations, the matrices $\mathbf{B}_{i, j}$ in (25) are generally different than the corresponding matrices in 24).

Using the technique presented in [36], we determine that, for any parity-check matrix in the form of (23), we can ensure

- $g \geq 6$ if all of the 18 matrices in the following set do not have a fixed column:

$\{\mathbf{P}, \mathbf{Q}, \mathbf{R}, \mathbf{S}, \mathbf{T}, \mathbf{U}$,

$$
\begin{aligned}
& \mathbf{P Q}^{\top}, \mathbf{P R}^{\top}, \mathbf{P T}^{\top}, \mathbf{Q S}^{\top}, \mathbf{Q U}^{\top}, \mathbf{R S}^{\top}, \mathbf{R T}^{\top}, \mathbf{S U}^{\top}, \mathbf{T U}^{\top}, \\
& \left.\mathbf{P Q}^{\top} \mathbf{S R}^{\top}, \mathbf{P Q}^{\top} \mathbf{U T}^{\top}, \mathbf{R} \mathbf{S}^{\top} \mathbf{U T}^{\top}\right\} ;
\end{aligned}
$$

- $g \geq 8$ if all of the 42 matrices in the following set do not have a fixed column:

$$
\begin{aligned}
& \{\mathbf{P}, \mathbf{Q}, \mathbf{R}, \mathbf{S}, \mathbf{T}, \mathbf{U}, \\
& \mathbf{P Q}^{\top}, \mathbf{P R}^{\top}, \mathbf{P S}^{\top}, \mathbf{P T}^{\top}, \mathbf{P U}^{\top}, \mathbf{Q R}^{\top}, \mathbf{Q S}^{\top}, \mathbf{Q T}^{\top}, \mathbf{Q U}^{\top}, \\
& \mathbf{R S}^{\top}, \mathbf{R T}^{\top}, \mathbf{R U}^{\top}, \mathbf{S T}^{\top}, \mathbf{S U}^{\top}, \mathbf{T U}^{\top}, \\
& \mathbf{P S R}^{\top}, \mathbf{P U T}^{\top}, \mathbf{R} \mathbf{T}^{\top}, \mathbf{T S R}^{\top}, \mathbf{R Q P}^{\top}, \mathbf{T Q P}^{\top}, \\
& \mathbf{R S}^{\top} \mathbf{Q}, \mathbf{R} \mathbf{S}^{\top} \mathbf{U}^{\top} \mathbf{T U}^{\top} \mathbf{Q}, \mathbf{T U}^{\top} \mathbf{S}, \mathbf{P Q}^{\top} \mathbf{S}, \mathbf{P Q}^{\top} \mathbf{U}^{\top}, \\
& \mathbf{P Q}^{\top} \mathbf{S R}^{\top}, \mathbf{P Q}^{\top} \mathbf{S T}^{\top}, \mathbf{P Q}^{\top} \mathbf{U R}^{\top}, \mathbf{P Q}^{\top} \mathbf{U T}^{\top}, \mathbf{P S}^{\top} \mathbf{U T}^{\top}, \\
& \left.\mathbf{R Q}^{\top} \mathbf{U T}^{\top}, \mathbf{R S}^{\top} \mathbf{Q T}^{\top}, \mathbf{R S}^{\top} \mathbf{U P}^{\top}, \mathbf{R S}^{\top} \mathbf{U T}^{\top}\right\} .
\end{aligned}
$$

Following the same process, additional conditions can be used to guarantee even larger girths. We will see later that, by pre-lifting $\mathbf{B}$ to $\mathbf{B}^{\uparrow m}$, the number of such conditions that must be checked in order to achieve girth $g$ for a derived matrix $\mathbf{H}=\mathbf{B}^{\uparrow m \circlearrowleft r} \in \xi_{\mathbf{B}^{\uparrow m}}(r)$ can be significantly less than for a general lifted matrix $\mathbf{H}=\mathbf{B}^{\uparrow m r} \in \xi_{\mathbf{B}}(m r)$. Moreover, we can use the circulant-based structure and corresponding modular arithmetic to reduce the complexity of evaluating the conditions and searching for suitable permutation matrices.

Recall that, if we take a direct circulant-based lifting of $\mathbf{B}$, the existence of a 4- or 6-cycle in the Tanner graph automatically implies a codeword of weight less than the upper bound $d_{\min } \leq 24$, so a minimum girth of 8 is required to achieve the bound. The $[124,33,24]$ QC Tanner code defined in (5) is an example of a code achieving the upper bound with girth $g=8$. In the remainder of this section we show that, by pre-lifting the $3 \times 4$ all-ones base matrix $\mathbf{B}$, we can construct circulant-based codes with minimum distance exceeding the upper bound $d_{\min } \leq 24$ for $\mathrm{QC}$ codes drawn from $\xi_{\mathrm{B}}^{Q C}(N)$, even if a 6 -cycle exists in the graph. Moreover, we observe further improvements by ensuring a girth larger than 6 .
From (24), there are $m^{6}=64$ possible 2 -covers of $\mathbf{B}$ that can be considered as candidates $\mathbf{B}^{\uparrow 2}$ for the pre-lifting step. After removing equivalent covering graphs (the 2-covers that are equal after re-labeling the vertices) there are five candidates left. Note that the only harmful substructure to avoid in a 2cover is the single occurrence of two disjoint 1 -covers. This can only occur if $\mathbf{B}_{i, j}=\mathbf{I}_{0}^{m}$ for all $(i, j) \in\{2,3\} \times\{2,3,4\}$. Any code $\mathcal{C}$ drawn from this QC sub-ensemble $\xi_{\mathbf{B}^{\uparrow 2}}^{Q C}(r)$ will have minimum distance bounded above by $d_{\min } \leq 24$ for arbitrarily large $r$. After removing this 2-cover, we have only four remaining candidates. Of these candidates, two give $d_{\min } \leq 120$ and two give $d_{\min } \leq 116$, both significantly larger than the bound for the 1 -cover, $d_{\min } \leq 24$.

Example 4. Consider the following 2-cover of B

$$
\mathbf{B}^{\uparrow 2}=\left[\begin{array}{ll|ll|ll|ll}
1 & 0 & 1 & 0 & 1 & 0 & 1 & 0 \\
0 & 1 & 0 & 1 & 0 & 1 & 0 & 1 \\
\hline 1 & 0 & 1 & 0 & 0 & 1 & 0 & 1 \\
0 & 1 & 0 & 1 & 1 & 0 & 1 & 0 \\
\hline 1 & 0 & 0 & 1 & 1 & 0 & 1 & 0 \\
0 & 1 & 1 & 0 & 0 & 1 & 0 & 1
\end{array}\right],
$$

from which, without loss of generality, any lifted code in the ensemble $\xi_{\mathbf{B}}^{Q C}(r)$ has the parity-check matrix

$$
\begin{aligned}
\mathbf{H}=\mathbf{B}^{\uparrow 2 \circlearrowleft r} & =\left[\begin{array}{cc|cc|cc|cc}
\mathbf{I}_{0}^{r} & \mathbf{0} & \mathbf{I}_{0}^{r} & \mathbf{0} & \mathbf{I}_{0}^{r} & \mathbf{0} & \mathbf{I}_{0}^{r} & \mathbf{0} \\
\mathbf{0} & \mathbf{I}_{0}^{r} & \mathbf{0} & \mathbf{I}_{0}^{r} & \mathbf{0} & \mathbf{I}_{0}^{r} & \mathbf{0} & \mathbf{I}_{0}^{r} \\
\hline \mathbf{I}_{0}^{r} & \mathbf{0} & \mathbf{I}_{p_{1}}^{r} & \mathbf{0} & \mathbf{0} & \mathbf{I}_{r_{1}}^{r} & \mathbf{0} & \mathbf{I}_{t_{1}}^{r} \\
\mathbf{0} & \mathbf{I}_{0}^{r} & \mathbf{0} & \mathbf{I}_{p_{2}}^{r} & \mathbf{I}_{r_{2}}^{r} & \mathbf{0} & \mathbf{I}_{t_{2}}^{r} & \mathbf{0} \\
\hline \mathbf{I}_{0}^{r} & \mathbf{0} & \mathbf{0} & \mathbf{I}_{q_{1}}^{r} & \mathbf{I}_{s_{1}}^{r} & \mathbf{0} & \mathbf{I}_{u_{1}}^{r} & \mathbf{0} \\
\mathbf{0} & \mathbf{I}_{0}^{r} & \mathbf{I}_{q_{2}}^{r} & \mathbf{0} & \mathbf{0} & \mathbf{I}_{s_{2}}^{r} & \mathbf{0} & \mathbf{I}_{u_{2}}^{r}
\end{array}\right] \\
& =\left[\begin{array}{cccc}
\mathbf{I}_{0}^{2 r} & \mathbf{I}_{0}^{2 r} & \mathbf{I}_{0}^{2 r} & \mathbf{I}_{0}^{2 r} \\
\mathbf{I}_{0}^{2 r} & \mathbf{P} & \mathbf{R} & \mathbf{T} \\
\mathbf{I}_{0}^{2 r} & \mathbf{Q} & \mathbf{S} & \mathbf{U}
\end{array}\right] .
\end{aligned}
$$

Using (6), we find that codes drawn from $\xi_{\mathbf{B}^{\uparrow 2}}^{Q C}(r)$ have minimum distance bounded above by $d_{\min } \leq 116$. Note that, because we have a small lifting factor $m=2$, all of the permutations in $\mathbf{B}^{\uparrow 2}$ are circulant, i.e., every pair of submatrices commute. In this case we must use Design Rule 1 and make sure the circulants chosen at the second step allow the minimum distance to exceed 24.

We also wish to ensure that the Tanner graph has an acceptable girth. Recall that if the 18 matrices in 26) do not have a fixed column, then $g \geq 6$, and if the 42 matrices in 27) do not have a fixed column, then $g \geq 8$. By applying Lemma 6 with the circulant-block permutation matrices from (28), we find that the number of conditions from (26) and 27) that we need to check is reduced to 8 and 20 , respectively. As an example, one surviving condition is that

$$
\begin{aligned}
& \mathbf{R T}^{\top}=\left[\begin{array}{cc}
\mathbf{0} & \mathbf{I}_{r_{1}}^{r} \\
\mathbf{I}_{r_{1}}^{r} & \mathbf{0}
\end{array}\right]\left[\begin{array}{cc}
\mathbf{0} & \mathbf{I}_{t_{1}}^{r} \\
\mathbf{I}_{t_{2}}^{r} & \mathbf{0}
\end{array}\right]^{\top} \\
& =\left[\begin{array}{cc}
\mathbf{0} & \mathbf{I}_{r_{1}}^{r} \\
\mathbf{I}_{r_{1}}^{r} & \mathbf{0}
\end{array}\right]\left[\begin{array}{cc}
\mathbf{0} & \mathbf{I}_{r-t_{2}}^{r} \\
\mathbf{I}_{r-t_{1}}^{r} & \mathbf{0}
\end{array}\right]=\left[\begin{array}{cc}
\mathbf{I}_{r_{1}+r-t_{1}}^{r} & \mathbf{0} \\
\mathbf{0} & \mathbf{I}_{r_{1}+r-t_{2}}^{r}
\end{array}\right]
\end{aligned}
$$

should not have a fixed column. This can be achieved simply by ensuring that $r_{i}+r-t_{i} \not \equiv 0 \bmod r$, for $i=1,2$. 
- Choosing the shift parameters

$$
\begin{gathered}
\left(p_{1}, p_{2}, q_{1}, q_{2}, r_{1}, r_{2}, s_{1}, s_{2}, t_{1}, t_{2}, u_{1}, u_{2}\right)= \\
(1,5,7,3,2,10,14,6,4,20,28,9),
\end{gathered}
$$

results in circulant-block permutation matrices $\mathbf{P}, \mathbf{Q}$, $\mathbf{R}, \mathbf{S}, \mathbf{T}, \mathbf{U}$ that give $g \geq 6$ for $r \geq 31$. For $r=31$, we find that $d_{\min }=36$ and $g=6$.

- By increasing the circulant size to $r=41$, we find that $g=6$ and determine that the minimum distance is bounded by $38 \leq d_{\min } \leq 48$ using MAGMA [39] 4 ]

- Recall that direct circulant liftings of $\mathbf{B}$ have minimum distance bounded above by $d_{\min } \leq 24$ for arbitrarily large circulant size, and a cycle of length 6 implies $d_{\min }<24$ (see [21]).

Example 5. Choosing the shift parameters in 29) to be

$$
\begin{gathered}
\left(p_{1}, p_{2}, q_{1}, q_{2}, r_{1}, r_{2}, s_{1}, s_{2}, t_{1}, t_{2}, u_{1}, u_{2}\right)= \\
(1,5,7,7,10,10,11,11,13,13,2,4),
\end{gathered}
$$

gives $g>6$ for $r \geq 20$. In fact, even for $r=17$, we obtain a $[136,36,26]$ code with $g=8$. By increasing the circulant size to $r=49$, the code has $g=10$ and we can determine that the minimum distance is bounded by $32 \leq d_{\min } \leq 56$ using MAGMA.

In this section, we have applied the techniques of prelifting to a $(3,4)$-regular protograph. We observed a large improvement in the minimum distance of QC-LDPC codes lifted from a 2-cover and we expect further improvement for larger pre-lifting factors $m$.

\section{Irregular protographs}

Luby et al. showed that the performance of LDPC codes can be improved significantly by introducing irregularity into the code graphs [40]. So far in this section we have only considered regular all-ones base matrices, but irregularities can easily be introduced by removing edges of the protograph. This technique, called masking, was introduced in [41] to construct good irregular LDPC codes from arrays of circulants. Masking involves replacing a number of the $N \times N$ permutation matrices with the $N \times N$ all-zero matrix. In particular, masking removes cycles in the graph and can increase the girth.

Example 6. Consider the masked (3,4)-regular base matrix $\mathbf{B}$ and its corresponding $N$-lifted parity-check matrix $\mathbf{H}=\mathbf{B}^{\uparrow N}$

$$
\mathbf{B}=\left[\begin{array}{llll}
1 & 1 & 1 & 1 \\
1 & 0 & 1 & 1 \\
1 & 1 & 1 & 0
\end{array}\right] \text { and } \mathbf{H}=\left[\begin{array}{cccc}
\mathbf{I}_{0}^{N} & \mathbf{I}_{0}^{N} & \mathbf{I}_{0}^{N} & \mathbf{I}_{0}^{N} \\
\mathbf{I}_{0}^{N} & \mathbf{0} & \mathbf{R} & \mathbf{T} \\
\mathbf{I}_{0}^{N} & \mathbf{Q} & \mathbf{S} & \mathbf{0}
\end{array}\right]
$$

\footnotetext{
${ }^{4}$ Due to the computational complexity, we are not able to determine the minimum distance of this example exactly. However, we conjecture that it is, in fact, equal or close to the upper bound based on the results obtained for smaller values of $r$ and the significant search time without finding any codewords of weight less than 48 .
}

Recall that, for the unmasked ensemble with parity-check matrix given in (23), in order to achieve girth $g \geq 8$ it was required that each of the 42 permutation matrices given in 27) should have no fixed columns. For the masked version of $\mathbf{H}$ in the form of 30], the number of permutation matrices that must be checked is reduced to these 13 :

$$
\begin{aligned}
& \left\{\mathbf{Q}, \mathbf{R}, \mathbf{S}, \mathbf{T}, \mathbf{Q S}^{\top}, \mathbf{R S}^{\top}, \mathbf{R T}^{\top},\right. \\
& \left.\mathbf{Q R}^{\top}, \mathbf{Q T}^{\top}, \mathbf{S T}^{\top}, \mathbf{R S}^{\top} \mathbf{Q}, \mathbf{T S R}^{\top}, \mathbf{R S}^{\top} \mathbf{Q T}^{\top}\right\},
\end{aligned}
$$

where only the first 7 should have no fixed columns to ensure $g \geq 6$. Moreover, suppose $\mathbf{B}$ is pre-lifted to $\mathbf{B}^{\uparrow 2}$ using the permutation matrices from 28). Then the number of permutation matrices in $\mathbf{H}=\mathbf{B}^{\uparrow 2 \circlearrowleft r}$ that must be checked is further reduced to these 6 :

$$
\left\{\mathbf{S}, \mathbf{R} \mathbf{T}^{\top}, \mathbf{Q R}^{\top}, \mathbf{Q T}{ }^{\top}, \mathbf{R S}^{\top} \mathbf{Q}, \mathbf{T S R}^{\top}\right\},
$$

with only the first two matrices needing to be checked to ensure $g \geq 6$.

Note that, while masking can improve the cycle properties of a Tanner graph, it often has a negative effect on minimum distance.

- For this example, we find that the upper bound on distance for any QC-LDPC code in $\xi_{\mathrm{B}}^{Q C}(N)$ with paritycheck matrix $\mathbf{H}=\mathbf{B}^{\circlearrowleft N}$ is reduced to $d_{\min } \leq 14$ (recall that for the unmasked case $d_{\min } \leq 24$ ).

- This dramatic decrease in minimum distance is likely a result of the large number of weight 2 columns in $\mathbf{H}$, and in this case pre-lifting is even more important 5

- We find that by pre-lifting the masked base matrix, the upper bound on minimum distance for masked pre-lifted QC-LDPC codes in $\xi_{\mathbf{B}^{\uparrow 2}}^{Q C}(r)$ with parity-check matrix $\mathbf{H}=\mathbf{B}^{\uparrow 2 \circlearrowleft r}$ is increased to $d_{\min } \leq 34$.

Remark 12 Note that very good irregular LDPC codes have been designed by optimizing their degree distribution [43]. Masking applied to a pre-lifted base matrix $\mathbf{B}^{\uparrow m}$ rather than the original base matrix $\mathbf{B}$ can give a code designer more flexibility in optimizing the degree distribution. In addition, optimizing degree distributions to improve performance in the waterfall region of the bit error rate (BER) curve often requires using many low-degree variable nodes [43]. In this case, prelifting can be used to good effect to mitigate the negative effect on minimum distance. This is particularly important for applications that require very low decoded BERs.

\section{Protographs with repeated edges}

In this section, we demonstrate the pre-lifting procedure applied to protographs with repeated edges. A great deal of effort has been devoted to designing protograph-based code ensembles with desirable features such as good iterative

${ }^{5}$ It is well known that the minimum distance properties of both unstructured [42] and protograph-based [6] code ensembles are sensitive to the number of degree two variable nodes in the code graph. In the case of structured code ensembles, such as protograph-based code ensembles, the connectivity of the degree two variable nodes is also important (see, e.g., [6]). 
decoding thresholds and linear minimum distance growth. These protographs typically have repeated edges [6], [7].

Example 7. The following $(3,4)$-regular example is taken from [21]. Consider the $3 \times 4$ base matrix

$$
\mathbf{B}=\left[\begin{array}{llll}
2 & 0 & 1 & 1 \\
1 & 1 & 2 & 0 \\
0 & 2 & 0 & 2
\end{array}\right]
$$

Note that $\mathbf{B}$ has some entries greater than 1; consequently, when lifting the corresponding protograph to form a paritycheck matrix $\mathbf{H}$, those entries $B_{i, j}$ are replaced by a summation of $B_{i, j}$ non-overlapping permutation matrices (or circulant permutation matrices if desired). It was shown in [21] that the upper bound on minimum distance obtained for any circulantbased lifting of this base matrix is $d_{\min } \leq 32$. Recall that for the $3 \times 4$ all-ones base matrix $\mathbf{B}$ we had $d_{\text {min }} \leq 24$, so the upper bound on minimum distance is improved by including repeated edges. Indeed, the following circulant-based lifting of $\mathbf{B}$

$\mathbf{H}=\mathbf{B}^{\circlearrowleft 46}=\left[\begin{array}{cccc}\mathbf{I}_{1}^{46}+\mathbf{I}_{2}^{46} & \mathbf{0} & \mathbf{I}_{4}^{46} & \mathbf{I}_{8}^{46} \\ \mathbf{I}_{5}^{46} & \mathbf{I}_{9}^{46} & \mathbf{I}_{10}^{46}+\mathbf{I}_{20}^{46} & \mathbf{0} \\ \mathbf{0} & \mathbf{I}_{25}^{46}+\mathbf{I}_{19}^{46} & \mathbf{0} & \mathbf{I}_{7}^{46}+\mathbf{I}_{14}^{46}\end{array}\right]$.

results in a $[184,47,32]$ code with girth $g=8$.

Now consider the following pre-lifted base matrix

$$
\mathbf{B}^{\uparrow 2}=\left[\begin{array}{cc|cc|cc|cc}
1 & 1 & 0 & 0 & 1 & 0 & 1 & 0 \\
1 & 1 & 0 & 0 & 0 & 1 & 0 & 1 \\
\hline 1 & 0 & 1 & 0 & 1 & 1 & 0 & 0 \\
0 & 1 & 0 & 1 & 1 & 1 & 0 & 0 \\
\hline 0 & 0 & 1 & 1 & 0 & 0 & 1 & 1 \\
0 & 0 & 1 & 1 & 0 & 0 & 1 & 1
\end{array}\right]
$$

As a result of pre-lifting, the minimum distance of a code drawn from $\xi_{\mathbf{B} \uparrow 2}^{Q C}(r)$ with parity-check matrix $\mathbf{H}=\mathbf{B}^{\uparrow 2 \circlearrowleft r}$ is bounded above by $d_{\min } \leq 108$, significantly larger than the upper bound $d_{\min } \leq 32$ obtained for codes drawn from $\xi_{\mathbf{B}}^{Q C}(N)$ with parity-check matrix $\mathbf{H}=\mathbf{B}^{\circlearrowleft N}$ An example of a code with minimum distance exceeding the original bound is the null space of the following parity-check matrix

$\mathbf{H}=\mathbf{B}^{\uparrow 2 \circlearrowleft 46}=\left[\begin{array}{cc|cc|cc|cc}\mathbf{I}_{1}^{46} & \mathbf{I}_{2}^{46} & 0 & 0 & \mathbf{I}_{4}^{46} & 0 & \mathbf{I}_{8}^{46} & 0 \\ \mathbf{I}_{2}^{46} & \mathbf{I}_{0}^{46} & 0 & 0 & 0 & \mathbf{I}_{4}^{46} & 0 & \mathbf{I}_{8}^{46} \\ \hline \mathbf{I}_{5}^{46} & 0 & \mathbf{I}_{9}^{46} & 0 & \mathbf{I}_{10}^{46} & \mathbf{I}_{20}^{46} & 0 & 0 \\ 0 & \mathbf{I}_{5}^{46} & 0 & \mathbf{I}_{9}^{46} & \mathbf{I}_{20}^{46} & \mathbf{I}_{10}^{46} & 0 & 0 \\ \hline 0 & 0 & \mathbf{I}_{25}^{46} & \mathbf{I}_{19}^{46} & 0 & 0 & \mathbf{I}_{7}^{46} & \mathbf{I}_{14}^{46} \\ 0 & 0 & \mathbf{I}_{19}^{46} & \mathbf{I}_{25}^{46} & 0 & 0 & \mathbf{I}_{14}^{46} & \mathbf{I}_{7}^{46}\end{array}\right]$.

This matrix defines a $[368,93,56]$ code with girth $g=8$, i.e., the minimum distance is significantly larger than the upper bound of 32 for any circulant-based 1-cover $\mathbf{H}=\mathbf{B}^{\circlearrowleft 2 r}$. Note that the same design rules must be applied to protographs with repeated edges. In this example, the circulant-block matrix $\mathbf{H}_{1,1}$ in the upper-left corner ensures that the submatrices do

\footnotetext{
${ }^{6}$ If $B_{i, j}>1$, it is not required that the corresponding $B_{i, j}$ permutation matrices selected at the pre-lifting step are non-overlapping; however, this condition must be enforced at the second lifting step.
}

not all commute and thus the upper bound can be exceeded. If, for example, we set

$$
\mathbf{H}_{1,1}=\left[\begin{array}{ll}
\mathbf{I}_{1}^{46} & \mathbf{I}_{2}^{46} \\
\mathbf{I}_{2}^{46} & \mathbf{I}_{1}^{46}
\end{array}\right]
$$

then all the submatrices would commute and we would have $d_{\min } \leq 32$.

\section{Code Design Rule 1: Pre-Lifting With CIRCULANT PERMUTATION MATRICES}

In this section, we focus on Design Rule 1, where the pre-lifted protograph $\mathbf{B}^{\uparrow m}$ is comprised of commuting submatrices. In particular, we consider the case when all of the permutation matrices $\mathbf{B}_{i, j}$ comprising $\mathbf{B}^{\uparrow m}$ are circulant. By choosing the permutations at the pre-lifting step to be circulant, we can make use of their structure to eliminate many of the conditions that must otherwise be checked to achieve a desired girth $g$. Moreover, the conditions can be evaluated efficiently using modular arithmetic.

\section{A. Girth conditions}

As noted previously, the technique given in [36] can be used to generate a set of conditions on the permutation matrices comprising $\mathbf{H}=\mathbf{B}^{\uparrow N}$ that must be satisfied to guarantee a girth of at least $g$. Now consider a parity-check matrix $\mathbf{H}=\mathbf{B}^{\uparrow m \circlearrowleft r}$, where $N=m r$ and $m$ is the pre-lifting factor. By applying Lemma 6 to $\mathbf{H}$, we can eliminate many of the conditions that must be satisfied by a general matrix $\mathbf{H}=\mathbf{B}^{\uparrow m r}$ by checking if the corresponding products of the associated permutation matrices $\mathbf{B}_{i, j}$ comprising $\mathbf{B}^{\uparrow m}$ have fixed columns. Choosing circulant permutation matrices at the pre-lifting step is advantageous for this purpose because we can quickly determine if a product of a number of circulant matrices has a fixed column using simple modular arithmetic (rather than costly matrix multiplication). This allows us to construct pre-lifted base matrices that reduce the number of conditions that must be satisfied in order to guarantee girth $g$ in Step 2 of the code design process.

Example 8. In this example, we focus on achieving $g \geq 8$ for a parity-check matrix in the form of 23, derived from a prelifted base matrix, but the same principles can be applied to a general protograph-based parity-check matrix derived from a pre-lifted base matrix for any desired girth. Suppose that

$$
\mathbf{P}=\operatorname{diag}\left(\mathbf{I}_{p_{1}}^{r}, \mathbf{I}_{p_{2}}^{r}, \ldots, \mathbf{I}_{p_{m}}^{r}\right) \cdot \tilde{\mathbf{I}}_{p}^{m},
$$

where $p \in[m]$ and $p_{i} \in[r]$, i.e., circulant-block matrix $\mathbf{P}$ is obtained by a double circulant-based lifting. (Similar definitions apply to $\mathbf{Q}, \mathbf{R}, \mathbf{S}, \mathbf{T}$, and $\mathbf{U}$.)

- For pre-lifting factor $m=5$ and any pre-lifted base matrix $\mathbf{B}^{\circlearrowleft 5}$ obtained using circulant submatrices, the number of conditions (from the set 27) on the permutation matrices that comprise $\mathbf{H}=\mathbf{B}^{\circlearrowleft 5 \circlearrowleft r}$ that must be checked to guarantee $g \geq 8$ is in the range $[4,42]$. 
- Consider the following pre-lifted base matrix $\mathbf{B}^{\uparrow m}$ in the form of (24) with $m=5$ :

$$
\mathbf{B}^{\circlearrowleft 5}=\left[\begin{array}{rrrr}
\mathbf{I}_{0}^{5} & \mathbf{I}_{0}^{5} & \mathbf{I}_{0}^{5} & \mathbf{I}_{0}^{5} \\
\mathbf{I}_{0}^{5} & \mathbf{I}_{0}^{5} & \mathbf{I}_{1}^{5} & \mathbf{I}_{1}^{5} \\
\mathbf{I}_{0}^{5} & \mathbf{I}_{0}^{5} & \mathbf{I}_{2}^{5} & \mathbf{I}_{4}^{5}
\end{array}\right] .
$$

By choosing the permutation matrices given above at the pre-lifting step, we find that, in order to guarantee $g \geq$ 8 in any resulting parity-check matrix $\mathbf{H}=\mathbf{B}^{\circlearrowleft 5 \circlearrowleft r} \epsilon$ $\xi_{\mathbf{B}^{\uparrow m}}^{Q C}(r)$, out of the 42 original conditions given in [27, we only need to check that $\mathbf{P}, \mathbf{Q}, \mathbf{P Q}^{\top}$, and $\mathbf{R} \mathbf{T}^{\top}$ do not have a fixed column. Equivalently, we must ensure $p_{i} \not \equiv 0$ $\bmod r, q_{i} \not \equiv 0 \bmod r, p_{i}+\left(r-q_{i}\right) \equiv p_{i}-q_{i} \not \equiv 0$ $\bmod r$, and $r_{i}-t_{i} \not \equiv 0 \bmod r, i=1,2, \ldots, 5$. Since $\mathbf{S}$ and $\mathbf{U}$ are not involved in these four conditions, the values $s_{i}, u_{i}, i=1, \ldots, 5$ can be chosen arbitrarily.

- In order to eliminate all the conditions given in 27), it is necessary to increase the pre-lifting factor to $m=9$. Then we find that it is possible to construct a pre-lifted base matrix $\mathbf{B}^{\uparrow m}$ with circulant submatrices that has girth 8 . Consequently, by Corollary 5 any $\mathbf{H} \in \xi_{\mathbf{B} \uparrow m}^{Q C}(r)$ satisfies $g \geq 8$, i.e., there are no conditions on the matrices $\mathbf{P}, \mathbf{Q}$, $\mathbf{R}, \mathbf{S}, \mathbf{T}$, and $\mathbf{U}$ that must be satisfied, so $p_{i}, q_{i}, r_{i}, s_{i}, t_{i}$, and $u_{i}, i=1,2, \ldots, 9$, can be chosen arbitrarily and we always obtain $g \geq 8$. The following pre-lifted base matrix is one such example:

$$
\mathbf{B}^{\circlearrowleft 9}=\left[\begin{array}{rrrr}
\mathbf{I}_{0}^{9} & \mathbf{I}_{0}^{9} & \mathbf{I}_{0}^{9} & \mathbf{I}_{0}^{9} \\
\mathbf{I}_{0}^{9} & \mathbf{I}_{1}^{9} & \mathbf{I}_{3}^{9} & \mathbf{I}_{4}^{9} \\
\mathbf{I}_{0}^{9} & \mathbf{I}_{2}^{9} & \mathbf{I}_{6}^{9} & \mathbf{I}_{8}^{9}
\end{array}\right] .
$$

\section{B. Minimum distance properties}

In this section, we construct a code using a circulant-based pre-lifting and show how its minimum distance is affected if we do not satisfy the overlapping column condition in Design Rule 1.

Example 9. Consider the pre-lifted base matrix $\mathbf{B}^{\uparrow 2}$ given in (28) with $m=2$ and the lifted parity-check matrix $\mathbf{H} \in \xi_{\mathbf{B} \uparrow m}^{Q C}(r)$ given in 29). Suppose that we set $p_{1}=p_{2}=1$, $q_{1}=q_{2}=7, r_{1}=r_{2}=10, s_{1}=s_{2}=11, t_{1}=t_{2}=13$, and $u_{1}=u_{2}=2$ with $r=49$. This parity-check matrix satisfies the conditions to achieve $g=10$. However, because the shift parameters in each circulant-block matrix are identical, this construction does not satisfy Design Rule 1. In fact, the conditions of Theorem 8 are satisfied, and the minimum distance is bounded above by $d_{\min } \leq\left(n_{c}+1\right) !=24$. This is in fact a $[392,100,24]$ QC code, i.e., the upper bound is achieved.

Suppose instead that we set $p_{2}=5$ and $u_{2}=4$, as in Example 5, and denote the resulting code $\mathcal{C}_{1}$. Then the circulant-block permutation matrices $\mathbf{P}$ and $\mathbf{U}$ are comprised of two different circulant submatrices and, consequently, there exists a pair of strongly noncommutative matrices (e.g., PQ and QP for $r=49)$, i.e., the conditions of Design Rule 1 are met. The minimum distance of $\mathcal{C}_{1}$ is increased to within the range $32 \leq d_{\min } \leq 56$ (determined using MAGMA) and $g=10$.

\section{Simulation results}

Computer simulations were performed assuming binary phase shift keyed (BPSK) modulation and an additive white Gaussian noise (AWGN) channel. The sum-product message passing decoder was allowed a maximum of 100 iterations and employed a syndrome-check based stopping rule. In Fig. 2. we plot the simulated decoding performance in terms of bit error rate $(\mathrm{BER})$ and frame error rate (FER) for: the prelifted $(3,4)$-regular QC code $\mathcal{C}_{1}$ with $m=2$ from Example 9 the extended $(3,4)$-regular QC Tanner code with parity-check matrix defined in (5), denoted by $\mathcal{C}_{2}$, where the circulant size is taken to be $N=98$ so that the code length and rate are the same as for code $\mathcal{C}_{1}$; and the original $(3,4)$-regular $\mathrm{QC}$ Tanner code with circulant size $N=31$, denoted by $\mathcal{C}_{3}$. Both codes $\mathcal{C}_{2}$ and $\mathcal{C}_{3}$ achieve the upper bound $d_{\text {min }}=24$ and have $g=8$. We observe that the pre-lifted code $\mathcal{C}_{1}$ has significantly improved decoding performance, with a signal-to-noise ratio (SNR) gain of over $1 \mathrm{~dB}$ at a bit error rate of $10^{-5}$. Moreover, we also see from Fig. 2, that the pre-lifted code outperforms a randomly constructed $(3,4)$-regular code of the same length and slightly lower rate, particularly at high SNRs.

When sub-optimal decoding methods are employed, there are many factors in addition to the girth and minimum distance of a code that affect its performance (such as pseudocodewords, trapping sets, and absorbing sets). Consequently, the improved simulated decoding performance of pre-lifted codes suggest pre-lifting may also improve these parameters.

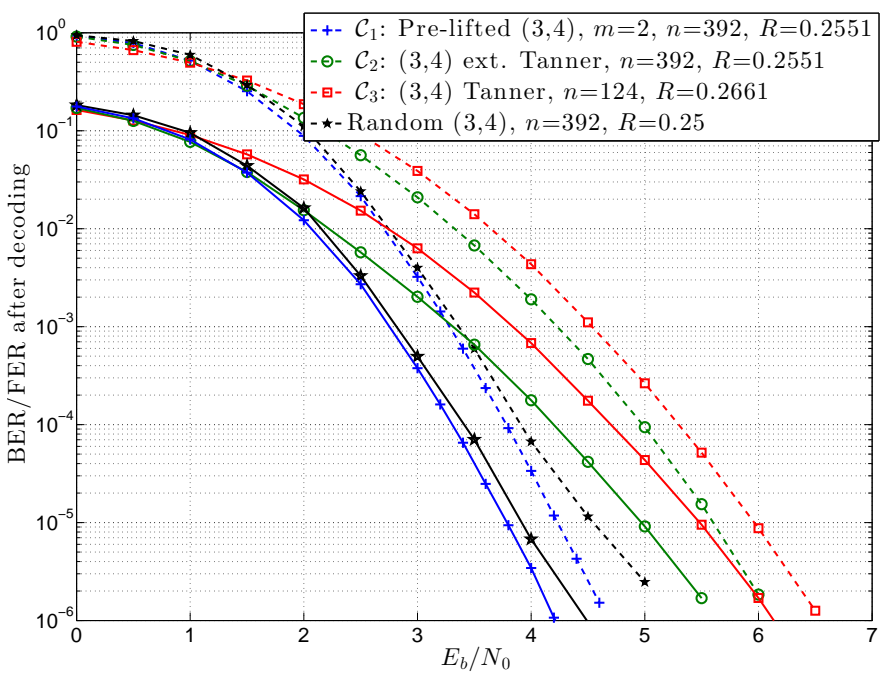

Fig. 2. Simulated decoding performance in terms of BER (solid lines) and FER (dashed lines) for the pre-lifted $(3,4)$-regular QC-LDPC code $\mathcal{C}_{1}$ described in Example 9 the extended $(3,4)$-regular Tanner QC-LDPC code $\mathcal{C}_{2}$, the original Tanner code $\mathcal{C}_{3}$, and a randomly constructed $(3,4)$-regular code. 


\section{Vi. Code Design Rule 2: Pre-lifting with NON-COMMUTING PERMUTATION MATRICES}

In this section, we construct a pre-lifted QC-LDPC code following Design Rule 2.

Example 10. We construct a parity-check matrix $\mathbf{H}$ derived from a pre-lifted base matrix $\mathbf{B}^{\uparrow m}$ defined in 24 with $m=4$. This matrix has the general form of (23), where $N=r m$ and

$$
\begin{aligned}
& {\left[\begin{array}{ccc}
\mathbf{P} & \mathbf{R} & \mathbf{T} \\
\mathbf{Q} & \mathbf{S} & \mathbf{U}
\end{array}\right]=\left[\begin{array}{ccc}
\mathbf{B}_{2,2} \otimes \mathbf{I}_{4}^{r} & \mathbf{B}_{2,3} \otimes \mathbf{I}_{12}^{r} & \mathbf{B}_{2,4} \otimes \mathbf{I}_{28}^{r} \\
\mathbf{B}_{3,2} \otimes \mathbf{I}_{24}^{r} & \mathbf{B}_{3,3} \otimes \mathbf{I}_{10}^{r} & \mathbf{B}_{3,4} \otimes \mathbf{I}_{13}^{r}
\end{array}\right]} \\
& =\left[\begin{array}{cccc|cccc|cccc}
\mathbf{0} & \mathbf{I}_{4}^{r} & \mathbf{0} & \mathbf{0} & \mathbf{0} & \mathbf{0} & \mathbf{I}_{12}^{r} & \mathbf{0} & \mathbf{0} & \mathbf{I}_{28}^{r} & \mathbf{0} & \mathbf{0} \\
\mathbf{I}_{4}^{r} & \mathbf{0} & \mathbf{0} & \mathbf{0} & \mathbf{0} & \mathbf{0} & \mathbf{0} & \mathbf{I}_{12}^{r} & \mathbf{0} & \mathbf{0} & \mathbf{I}_{28}^{r} & \mathbf{0} \\
\mathbf{0} & \mathbf{0} & \mathbf{0} & \mathbf{I}_{4}^{r} & \mathbf{I}_{12}^{r} & \mathbf{0} & \mathbf{0} & \mathbf{0} & \mathbf{0} & \mathbf{0} & \mathbf{0} & \mathbf{I}_{28}^{r} \\
\mathbf{0} & \mathbf{0} & \mathbf{I}_{4}^{r} & \mathbf{0} & \mathbf{0} & \mathbf{I}_{12}^{r} & \mathbf{0} & \mathbf{0} & \mathbf{I}_{28}^{r} & \mathbf{0} & \mathbf{0} & \mathbf{0} \\
\hline \mathbf{0} & \mathbf{0} & \mathbf{I}_{24}^{r} & \mathbf{0} & \mathbf{0} & \mathbf{I}_{10}^{r} & \mathbf{0} & \mathbf{0} & \mathbf{0} & \mathbf{0} & \mathbf{I}_{13}^{r} & \mathbf{0} \\
\mathbf{0} & \mathbf{0} & \mathbf{0} & \mathbf{I}_{24}^{r} & \mathbf{I}_{10}^{r} & \mathbf{0} & \mathbf{0} & \mathbf{0} & \mathbf{0} & \mathbf{0} & \mathbf{0} & \mathbf{I}_{13}^{r} \\
\mathbf{0} & \mathbf{I}_{24}^{r} & \mathbf{0} & \mathbf{0} & \mathbf{0} & \mathbf{0} & \mathbf{0} & \mathbf{I}_{10}^{r} & \mathbf{I}_{13}^{r} & \mathbf{0} & \mathbf{0} & \mathbf{0} \\
\mathbf{I}_{24}^{r} & \mathbf{0} & \mathbf{0} & \mathbf{0} & \mathbf{0} & \mathbf{0} & \mathbf{I}_{10}^{r} & \mathbf{0} & \mathbf{0} & \mathbf{I}_{13}^{r} & \mathbf{0} & \mathbf{0}
\end{array}\right] .
\end{aligned}
$$

Note that the pre-lifting permutation matrices $\mathbf{B}_{2,2}, \mathbf{B}_{3,2}$, $\mathbf{B}_{2,3}, \mathbf{B}_{3,3}, \mathbf{B}_{2,4}$, and $\mathbf{B}_{3,4}$ have been chosen so that several pairs of permutation matrices are strongly noncommutative, yet $\mathbf{B}_{2,3}, \mathbf{B}_{2,4}$, and $\mathbf{B}_{3,4}$ are, in fact, circulant. The pre-lifting permutation matrices were chosen following the techniques presented in Section IV in order to obtain large upper bounds on minimum distance. The shift parameters for each circulantblock permutation matrix were selected following the Tanner construction. Consequently, the pre-lifted Tanner graph associated with $\mathbf{H}$ can be considered as a 4-fold graph cover of the original Tanner graph.

For $r=14$, we obtain a $[224,59,36]$ QC-LDPC code with $g=8$. As we increase $r$, the minimum distance generally improves, but it is difficult to verify the exact value using MAGMA as the code length increases. For $r=31$, we obtain a $[496,126]$ QC-LDPC code, denoted by $\mathcal{C}_{4}$, with $g=8$ and $28 \leq d_{\min } \leq 68$ (as in Example 4, we conjecture that the minimum distance is, in fact, close to 68).

In Fig. 3, we show the decoding performance of $\mathcal{C}_{4}$ and two $(3,4)$-regular QC Tanner codes: the extended $(3,4)$-regular QC Tanner code, denoted by $\mathcal{C}_{5}$, defined in (5), where the circulant size is taken to be $N=124$ so that the rate is approximately equal to that of $\mathcal{C}_{4}$ and the code lengths are equal; and the original $(3,4)$-regular QC Tanner code $\mathcal{C}_{3}$ with $N=31$. Again, we observe significantly improved decoding performance for the pre-lifted QC code. Moreover, we see that it performs slightly better than a randomly constructed $(3,4)$-regular code of the same length and slightly lower rate, particularly at high SNRs.

Design Rule 2 is particularly useful because we can employ the theory presented in Section IV to design a good pre-lifting matrix and use state-of-the-art QC codes, like the Tanner codes, to choose the circulants at Step 2 of the code design procedure. In the next section, we will see that large gains in decoding performance can be achieved by pre-lifting a 'good' code.

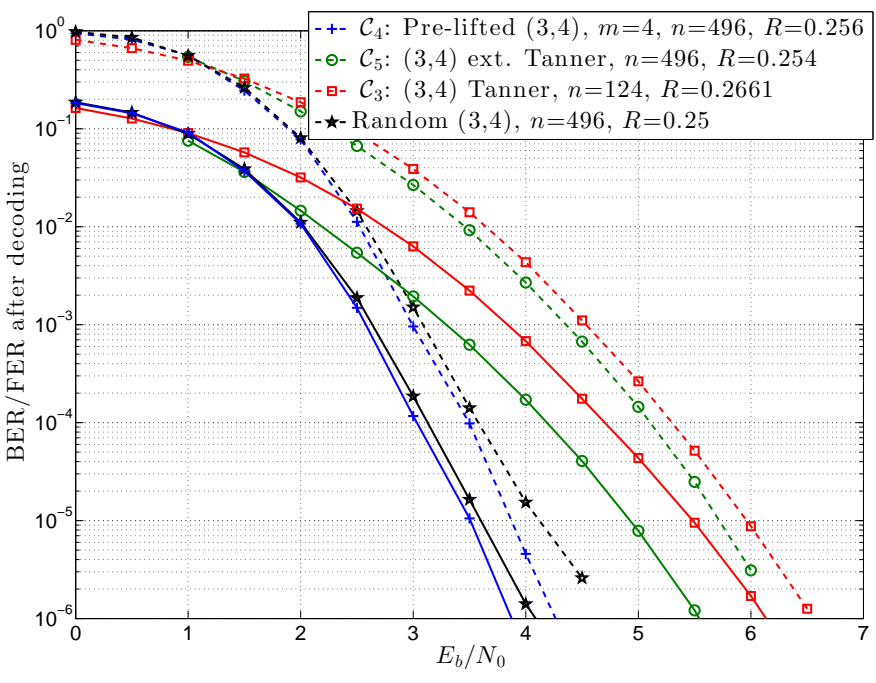

Fig. 3. Simulated decoding performance in terms of BER (solid lines) and FER (dashed lines) for the pre-lifted $(3,4)$-regular QC-LDPC code $\mathcal{C}_{4}$ described in Example 10 the extended $(3,4)$-regular Tanner QC-LDPC code $\mathcal{C}_{5}$, the original Tanner code $\mathcal{C}_{3}$, and a randomly constructed $(3,4)$-regular code.

\section{Code Design: Pre-Lifting 'Good’ Codes}

So far, we have used the Tanner code as a model, but Design Rule 2 can be applied to any array-based QC code. As a final example, we construct a nested family of QC-LDPC codes with design rates $R=1 / 4,2 / 5,1 / 2$, and $4 / 7$ using the prelifting technique. The model code we use is a $(3,7)$-regular QC-LDPC code with the following parity-check matrix [22]:

$$
\mathbf{H}=\mathbf{B}^{\circlearrowleft N}=\left[\begin{array}{ccccccc}
\mathbf{I}_{0}^{N} & \mathbf{I}_{19}^{N} & \mathbf{I}_{13}^{N} & \mathbf{I}_{20}^{N} & \mathbf{I}_{4}^{N} & \mathbf{I}_{15}^{N} & \mathbf{I}_{56}^{N} \\
\mathbf{I}_{18}^{N} & \mathbf{I}_{9}^{N} & \mathbf{I}_{0}^{N} & \mathbf{I}_{47}^{N} & \mathbf{I}_{0}^{N} & \mathbf{I}_{18}^{N} & \mathbf{I}_{8}^{N} \\
\mathbf{I}_{14}^{N} & \mathbf{I}_{0}^{N} & \mathbf{I}_{10}^{N} & \mathbf{I}_{13}^{N} & \mathbf{I}_{0}^{N} & \mathbf{I}_{0}^{N} & \mathbf{I}_{7}^{N}
\end{array}\right]_{,}
$$

which can be obtained by a one-step circulant-based lifting of the $3 \times 7$ all-ones base matrix B. The circulants in this matrix were carefully selected using so-called 'voltage graphs' in order to achieve a girth in the associated Tanner graph of 8 with lifting factor $N=111$. Moreover, this code achieves the upper bound on minimum distance of $d_{\min }=24$ for a direct circulant-based lifting of $\mathbf{B}$. Note that this construction is 'nested', in the sense that we can shorten this code to be $(3,4)-,(3,5)-,(3,6)-$, or $(3,7)$-regular by truncating (33) from the right to have $4,5,6$, or 7 (block) columns, and, for the lifting factor $N=111$, each code will achieve the upper bound of $d_{\min }=24$ for a direct circulant-based lifting of the corresponding (truncated) matrix $\mathbf{B}$. We will denote the code with $(3, K)$-regular parity-check matrix $\mathbf{H}$ and lifting factor $N$ by $\mathcal{C}_{6}(3, K, N)$.

In the following, we see that significantly improved decoding performance compared to this sequence of shortened codes can be obtained for each rate by carefully pre-lifting $\mathbf{B}$ and then using the same choice of circulants as in (33). In particular, we show empirically that the structural properties of the non-prelifted codes result in a 'limiting performance' and almost identical error floors as the lifting factor $N$ increases, whereas the pre-lifted codes exceed this performance with 
increasing $r$ as a result of their improved minimum distance.

Let $\mathbf{S}$ be the matrix of shift indices of $\mathbf{H}$, i.e.,

$$
\mathbf{S}=\left[\begin{array}{ccccccc}
0 & 19 & 13 & 20 & 4 & 15 & 56 \\
18 & 9 & 0 & 47 & 0 & 18 & 8 \\
14 & 0 & 10 & 13 & 0 & 0 & 7
\end{array}\right]
$$

Then the parity-check matrix of the $(3, K)$-regular QC-LDPC code, $K \in\{4,5,6,7\}$, based on the pre-lifting is given by

$$
\mathbf{H}=\left[\mathbf{H}_{i, j}\right]_{1 \leq i \leq 3 ; 1 \leq j \leq K}=\left[\mathbf{B}_{i, j} \otimes \mathbf{I}_{\mathbf{S}_{i, j}}^{r}\right]_{1 \leq i \leq 3 ; 1 \leq j \leq K},
$$

where the sub matrices $\mathbf{H}_{i, j}$ have size $m r \times m r$. We will denote the code with $(3, K)$-regular parity-check matrix $\mathbf{H}$ and lifting factor $r$ by $\mathcal{C}_{7}(3, K, m, r)$. Note that the girth of $\mathcal{C}_{7}(3, K, m, r)$ must be at least as large as the girth of $\mathcal{C}_{6}(3, K, r)$.

The pre-lifted matrix (36) is obtained from $\mathbf{B}$ with prelifting factor $m=4$, where the permutation matrices were chosen following the techniques presented in Section IV] to give large upper bounds on the minimum distance and, adhering to Design Rule 2, to ensure that at least one pair of submatrices $\left(\mathbf{B}_{i, j}, \mathbf{B}_{k, l}\right),(i, j) \neq(k, l)$, is strongly noncommutative.

Fig. 4 shows the decoding performance of $(3,4)$-regular QC-LDPC codes obtained for a variety of different lifting factors using circulant-based liftings of both the original base matrix $\mathbf{B}$ and the pre-lifted base matrix $\mathbf{B}^{\uparrow 4}$. We observe for the $\mathcal{C}_{6}(3,4, N)$ codes that, as $N$ increases, the performance at low to moderate SNR improves (we observe an approximately $0.5-0.7 \mathrm{~dB}$ gain in the BER range $10^{-2}$ to $10^{-4}$ by increasing $N$ from 111 to 222,444 , or 888 ); however, at high SNRs the codes all suffer from an error floor (the BERs for $N=111,222,444$, and 888 converge to approximately $2 \times 10^{-6}$ at an SNR of $\left.4 \mathrm{~dB}\right)$. The $\mathcal{C}_{6}(3,4, N)$ codes each have $g=10$.

For the pre-lifted QC-LDPC codes $\mathcal{C}_{7}(3,4,4, r)$, we observe significantly improved decoding performance; in particular, we do not observe any error floors down to a BER of $10^{-6}$ for $r=111,222$, and 444 , surpassing the 'limiting performance' of the QC codes derived directly from $\mathbf{B}$. The $\mathcal{C}_{7}(3,4,4, r)$ codes each have $g=10$ for these lifting factors. We also include the decoding performance for smaller lifting factors $r=28$ and $r=56$, even though the circulants were optimized in [22] for $N=111$. Consequently, these codes have reduced girth $g=6$; however, we see that, for $r=28$, the pre-lifted code $\mathcal{C}_{7}(3,4,4,28)$ has approximately the same decoding performance as $\mathcal{C}_{6}(3,4,111)$, illustrating that (36) represents a good choice for $\mathbf{B}^{\uparrow 4}$, and for $r=56$ we see improved performance in the high SNR region compared to any of the one-step liftings (even those with larger block lengths). Finally, we note that the performance of the pre-lifted code $\mathcal{C}_{7}(3,4,4,444)$ is only about $0.4 \mathrm{~dB}$ from the iterative decoding threshold $\gamma_{\text {iter }}=1.2758 \mathrm{~dB}$ of the $(3,4)$-regular protographbased ensemble $\xi_{\mathbf{B}}(N)$ at a BER of $10^{-6}$, and we would expect this gap to decrease as we increase $r 7$ Our results indicate that similar performance is unlikely to be realized for

\footnotetext{
${ }^{7}$ Iterative decoding thresholds for the AWGN channel were estimated using the reciprocal channel approximation (RCA) technique [44].
}

the $\mathcal{C}_{6}(3,4, N)$ codes, even by letting $N$ become very large, since these codes have limited minimum distance.

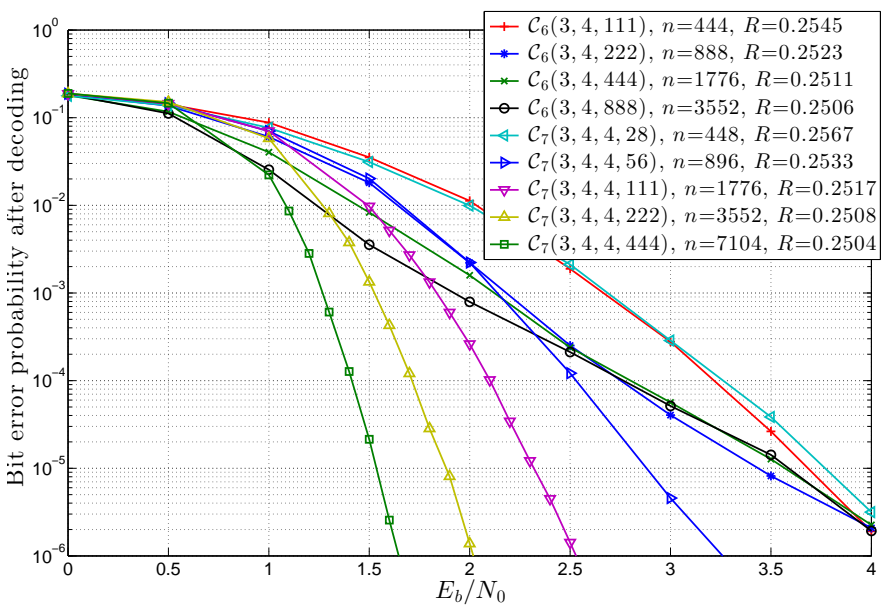

Fig. 4. Simulated decoding performance of several $(3,4)$-regular QCLDPC codes $\mathcal{C}_{6}(3,4, N)$ and the pre-lifted $(3,4)$-regular QC-LDPC codes $\mathcal{C}_{7}(3,4, m, r)$ for a variety of lifting factors.

Figures [5, 6] and 7 show the decoding performance of the higher rate $(3,5)-,(3,6)$-, and $(3,7)$-regular QC-LDPC codes, respectively, obtained for a variety of different lifting factors using circulant-based liftings of both the original base matrix $\mathbf{B}$ and the pre-lifted base matrix $\mathbf{B}^{\uparrow 4}$. We again see that the performance of the 4-covers with small lifting factor $r=28$ is approximately equal to the performance of the original code, indicating that (36) represents a good choice for $\mathbf{B}^{\uparrow 4}$ in each case. For each code rate, we see that the pre-lifted code $\mathcal{C}_{7}(J, K, 4,111)$ outperforms the once-lifted code $\mathcal{C}_{6}(J, K, 444)$ of the same length and approximately the same rate. Moreover, we expect that this gap will increase as we further increase the lifting factors. Finally, we note that the performance of the code $\mathcal{C}_{7}(3,6,4,111)$ in Fig. 6 is only slightly worse (about $0.1-0.2 \mathrm{~dB}$ ) than the $[2304,1152]$ WiMAX code [45], despite the fact that the construction described above involved only an easy search for a good prelifting matrix and then simply adopted (33) for the second lifting step.

We have seen that the 'limiting performance' of one-step circulant liftings of a code can be exceeded by a pre-lifted code. This result indicates that to design QC-LDPC codes with larger block lengths, it may be better to pre-lift the base matrix rather than increase the circulant size, since QCLDPC codes based on pre-lifted protographs have improved minimum distance and large girth. We attributed the improved decoding performance reported in Sections V VIII to these parameters; however, when sub-optimal iterative decoding methods are employed, there are other parameters in addition to girth and minimum distance that affect code performance (such as pseudocodewords, trapping sets, and absorbing sets). Consequently, the improved simulated decoding performance of pre-lifted codes suggests that pre-lifting may also improve these parameters. 


$\mathbf{B}^{\uparrow 4}=\left[\begin{array}{llll|llll|llll|lllll|llll|llll|lllll}1 & 0 & 0 & 0 & 1 & 0 & 0 & 0 & 1 & 0 & 0 & 0 & 1 & 0 & 0 & 0 & 1 & 0 & 0 & 0 & 1 & 0 & 0 & 0 & 1 & 0 & 0 & 0 \\ 0 & 1 & 0 & 0 & 0 & 1 & 0 & 0 & 0 & 1 & 0 & 0 & 0 & 1 & 0 & 0 & 0 & 1 & 0 & 0 & 0 & 1 & 0 & 0 & 0 & 1 & 0 & 0 \\ 0 & 0 & 1 & 0 & 0 & 0 & 1 & 0 & 0 & 0 & 1 & 0 & 0 & 0 & 1 & 0 & 0 & 0 & 1 & 0 & 0 & 0 & 1 & 0 & 0 & 0 & 1 & 0 \\ 0 & 0 & 0 & 1 & 0 & 0 & 0 & 1 & 0 & 0 & 0 & 1 & 0 & 0 & 0 & 1 & 0 & 0 & 0 & 1 & 0 & 0 & 0 & 1 & 0 & 0 & 0 & 1 \\ \hline 1 & 0 & 0 & 0 & 0 & 1 & 0 & 0 & 0 & 0 & 1 & 0 & 0 & 1 & 0 & 0 & 0 & 1 & 0 & 0 & 1 & 0 & 0 & 0 & 0 & 0 & 1 & 0 \\ 0 & 1 & 0 & 0 & 1 & 0 & 0 & 0 & 0 & 0 & 0 & 1 & 0 & 0 & 1 & 0 & 0 & 0 & 0 & 1 & 0 & 0 & 1 & 0 & 0 & 0 & 0 & 1 \\ 0 & 0 & 1 & 0 & 0 & 0 & 0 & 1 & 1 & 0 & 0 & 0 & 0 & 0 & 0 & 1 & 1 & 0 & 0 & 0 & 0 & 0 & 0 & 1 & 0 & 1 & 0 & 0 \\ 0 & 0 & 0 & 1 & 0 & 0 & 1 & 0 & 0 & 1 & 0 & 0 & 1 & 0 & 0 & 0 & 0 & 0 & 1 & 0 & 0 & 1 & 0 & 0 & 1 & 0 & 0 & 0 \\ \hline 1 & 0 & 0 & 0 & 0 & 0 & 1 & 0 & 0 & 1 & 0 & 0 & 0 & 0 & 1 & 0 & 0 & 0 & 1 & 0 & 0 & 1 & 0 & 0 & 1 & 0 & 0 & 0 \\ 0 & 1 & 0 & 0 & 0 & 0 & 0 & 1 & 1 & 0 & 0 & 0 & 0 & 0 & 0 & 1 & 1 & 0 & 0 & 0 & 0 & 0 & 1 & 0 & 0 & 0 & 1 & 0 \\ 0 & 0 & 1 & 0 & 0 & 1 & 0 & 0 & 0 & 0 & 0 & 1 & 1 & 0 & 0 & 0 & 0 & 0 & 0 & 1 & 1 & 0 & 0 & 0 & 0 & 0 & 0 & 1 \\ 0 & 0 & 0 & 1 & 1 & 0 & 0 & 0 & 0 & 0 & 1 & 0 & 0 & 1 & 0 & 0 & 0 & 1 & 0 & 0 & 0 & 0 & 0 & 1 & 0 & 1 & 0 & 0\end{array}\right]$,

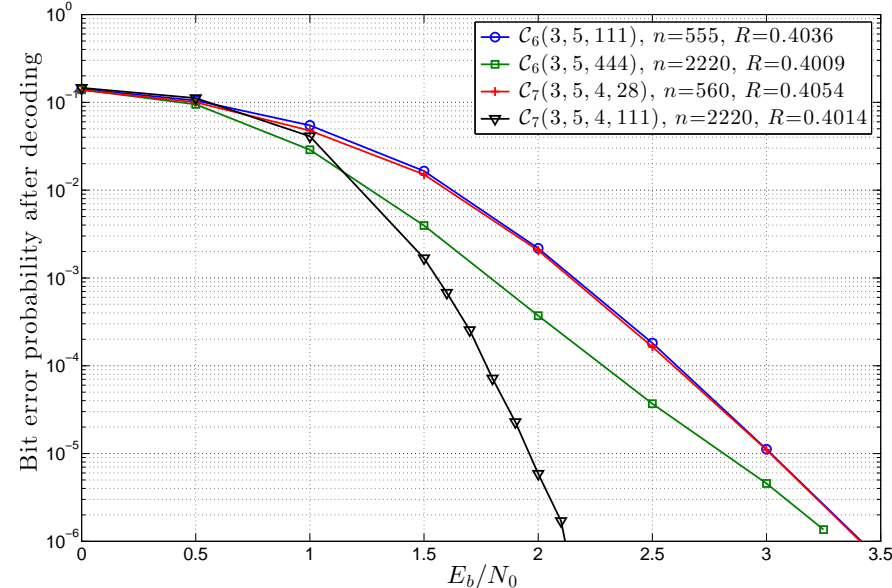

Fig. 5. Simulated decoding performance of several $(3,5)$-regular QCLDPC codes $\mathcal{C}_{6}(3,5, N)$ and the pre-lifted $(3,5)$-regular QC-LDPC codes $\mathcal{C}_{7}(3,5,4, r)$ for a variety of lifting factors.

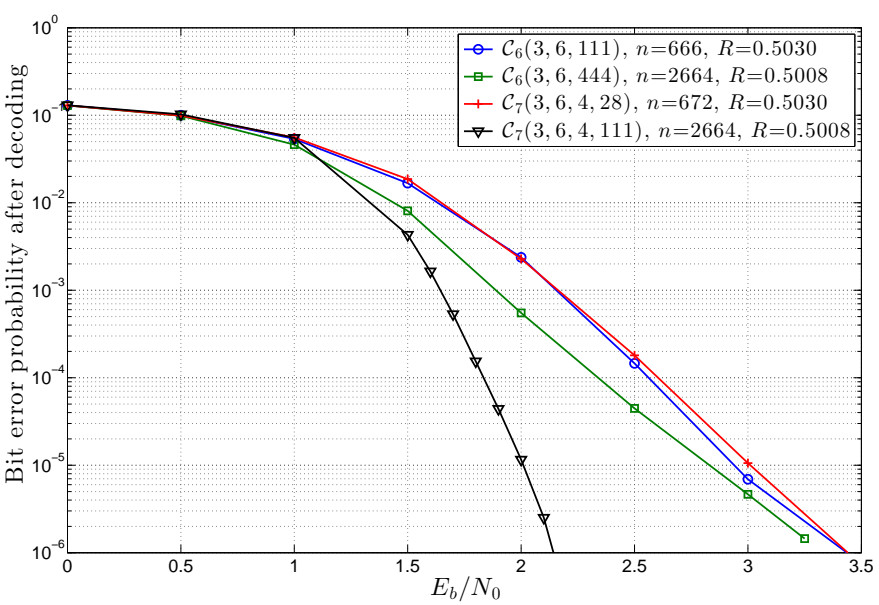

Fig. 6. Simulated decoding performance of several $(3,6)$-regular QCLDPC codes $\mathcal{C}_{6}(3,6, N)$ and the pre-lifted $(3,6)$-regular QC-LDPC codes $\mathcal{C}_{7}(3,6,4, r)$ for a variety of lifting factors.

\section{CONCLUDing REMARKS}

In this paper, we presented new results on QC-LDPC codes that are constructed using a two-step lifting procedure based on a protograph, and, by implementing this method instead of the usual one-step procedure, we were able to show improved minimum distance and girth properties. We also presented two design rules to construct QC-LDPC codes based on

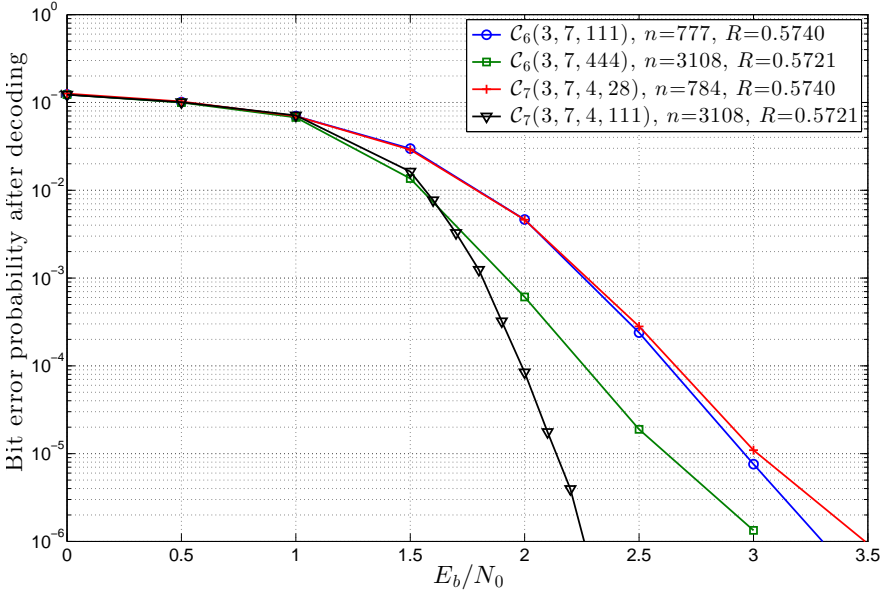

Fig. 7. Simulated decoding performance of several $(3,7)$-regular QCLDPC codes $\mathcal{C}_{6}(3,7, N)$ and the pre-lifted $(3,7)$-regular QC-LDPC codes $\mathcal{C}_{7}(3,7,4, r)$ for a variety of lifting factors.

pre-lifting: one uses only commuting pairs of permutation matrices at the first (pre-lifting) stage, while the other includes some strongly noncommutative pairs of permutation matrices. For both design rules, we obtained an increase in minimum distance compared to a one-step circulant-based lifting and improved performance was verified by simulation. Finally, we showed that the pre-lifting technique can also be applied to any 'good' QC-LDPC code existing in the literature and results in a new QC-LDPC code with improved minimum distance, girth, and decoding performance.

\section{APPENDIX A}

The matrix $\mathbf{H}=\mathbf{B}^{\uparrow N}$ consisting of an $n_{c} \times n_{v}$ array of permutation matrices $\mathbf{Q}_{i, j}, i \in\left\{1,2, \ldots, n_{c}\right\}, j \in\left\{1,2, \ldots, n_{v}\right\}$, can be transformed by column operations into

$$
\left[\begin{array}{cccc}
\mathbf{I}_{0}^{N} & \mathbf{I}_{0}^{N} & \cdots & \mathbf{I}_{0}^{N} \\
\mathbf{Q}_{2,1} \mathbf{Q}_{1,1}^{\top} & \mathbf{Q}_{2,2} \mathbf{Q}_{1,2}^{\top} & \cdots & \mathbf{Q}_{2, n_{v}} \mathbf{Q}_{1, n_{v}}^{\top} \\
\vdots & \vdots & & \vdots \\
\mathbf{Q}_{n_{c}, 1} \mathbf{Q}_{1,1}^{\top} & \mathbf{Q}_{n_{c}, 2} \mathbf{Q}_{1,2}^{\top} & \cdots & \mathbf{Q}_{n_{c}, n_{v}} \mathbf{Q}_{1, n_{v}}^{\top}
\end{array}\right],
$$

followed by row operations to transform it into

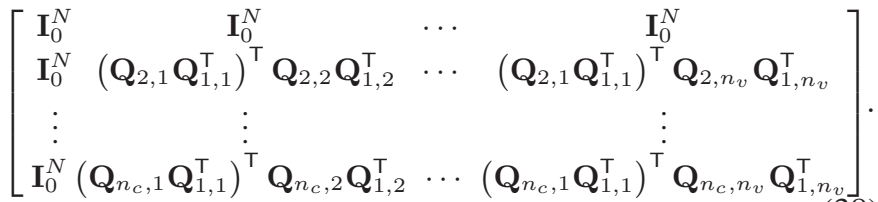


The operations described above do not affect the girth of the Tanner graph or the minimum distance of the code because we have simply reordered the rows and columns. If all permutation matrices $\mathbf{Q}_{i, j}$ are circulant (or circulant-block) then the products of such matrices as in 38 must also be circulant (resp. circulant-block) by Property 2 of circulant permutation matrices.

\section{APPENDIX B}

Proof of Lemma 6] Suppose $\mathbf{P}=\operatorname{diag}\left(\mathbf{I}_{p_{1}}^{r}, \mathbf{I}_{p_{2}}^{r}, \ldots, \mathbf{I}_{p_{m}}^{r}\right)$. $\tilde{\mathbf{B}}_{P}$ and $\mathbf{Q}=\operatorname{diag}\left(\mathbf{I}_{q_{1}}^{r}, \mathbf{I}_{q_{2}}^{r}, \ldots, \mathbf{I}_{q_{m}}^{r}\right) \cdot \tilde{\mathbf{B}}_{Q}$, so that

$\mathbf{P Q}=\operatorname{diag}\left(\mathbf{I}_{p_{1}}^{r}, \mathbf{I}_{p_{2}}^{r}, \ldots, \mathbf{I}_{p_{m}}^{r}\right) \tilde{\mathbf{B}}_{P} \cdot \operatorname{diag}\left(\mathbf{I}_{q_{1}}^{r}, \mathbf{I}_{q_{2}}^{r}, \ldots, \mathbf{I}_{q_{m}}^{r}\right) \tilde{\mathbf{B}}_{Q}$,

where $p_{i}, q_{i} \in[r], i \in[m], \mathbf{B}_{P}$ and $\mathbf{B}_{Q}$ are $m \times m$ permutation matrices, and $\tilde{\mathbf{B}}_{P}=\mathbf{B}_{P} \otimes \mathbf{I}_{0}^{r}$ (as defined in Section $\amalg$ - ). Then

$\mathbf{P Q}=$

$$
\begin{aligned}
& \operatorname{diag}\left(\mathbf{I}_{p_{1}}^{r}, \ldots, \mathbf{I}_{p_{m}}^{r}\right) \operatorname{diag}\left(\mathbf{I}_{q_{\sigma(1)}}^{r}, \ldots, \mathbf{I}_{q_{\sigma(m)}}^{r}\right) \cdot \tilde{\mathbf{B}}_{P} \tilde{\mathbf{B}}_{Q},= \\
& \operatorname{diag}\left(\mathbf{I}_{\left(p_{1}+q_{\sigma(1)}\right) \bmod r}^{r}, \ldots, \mathbf{I}_{\left(p_{m}+q_{\sigma(m)}\right) \bmod r}^{r}\right) \cdot \tilde{\mathbf{B}}_{P} \tilde{\mathbf{B}}_{Q},
\end{aligned}
$$

where $\sigma$ is the permutation associated with permutation matrix $\mathbf{B}_{P}$. Note also that by the distributive law of the Kronecker product

$$
\tilde{\mathbf{B}}_{P} \tilde{\mathbf{B}}_{Q}=\left(\mathbf{B}_{P} \otimes \mathbf{I}_{0}^{r}\right) \cdot\left(\mathbf{B}_{Q} \otimes \mathbf{I}_{0}^{r}\right)=\mathbf{B}_{P} \mathbf{B}_{Q} \otimes \mathbf{I}_{0}^{r} \mathbf{I}_{0}^{r} .
$$

Suppose $\mathbf{B}_{P} \mathbf{B}_{Q}$ does not have a fixed column, i.e., $\left(\mathbf{B}_{P} \mathbf{B}_{Q}\right)_{k, k}=0, k=1,2, \ldots, m$. Then $\left(\tilde{\mathbf{B}}_{P} \tilde{\mathbf{B}}_{Q}\right)_{i, j}=0$ for $(i, j) \in \mathcal{S}$, where

$$
\mathcal{S}=\bigcup_{k=1}^{m}\left(\mathcal{S}_{k} \times \mathcal{S}_{k}\right)=\bigcup_{k=1}^{m} \mathcal{S}_{k}^{2}
$$

$\mathcal{S}_{k}=\{(k-1) r+1,(k-1) r+2, \ldots, k r\}$, and $\mathcal{S}_{k} \times \mathcal{S}_{k}$ denotes the Cartesian product of two sets. Now,

$$
\begin{aligned}
& (\mathbf{P Q})_{i, i} \\
& =\sum_{j=1}^{m r}\left(\operatorname{diag}\left(\mathbf{I}_{\left(p_{1}+q_{\sigma(1)}\right)}^{r}, \ldots, \mathbf{I}_{\left(p_{m}+q_{\sigma(m)}\right)}^{r}\right)\right)_{i, j}\left(\tilde{\mathbf{B}}_{P} \tilde{\mathbf{B}}_{Q}\right)_{j, i},
\end{aligned}
$$

for $i \in\{1,2, \ldots, m r\}$. Suppose $i \in \mathcal{S}_{k}$; then, it follows from the structure of a block diagonal permutation matrix that the only non-zero symbol $\left(\operatorname{diag}\left(\mathbf{I}_{\left(p_{1}+q_{\sigma(1)}\right)}^{r}, \mathbf{I}_{\left(p_{2}+q_{\sigma(2)}\right)}^{r}, \ldots, \mathbf{I}_{\left(p_{m}+q_{\sigma(m)}\right)}^{r}\right)\right)_{i, j} \quad$ occurs when $j \in \mathcal{S}_{k}$. Then $(j, i) \in \mathcal{S}$, which implies $\left(\tilde{\mathbf{B}}_{P} \tilde{\mathbf{B}}_{Q}\right)_{j, i}=0$, and thus $(\mathbf{P Q})_{i, i}=0$ follows from (41).

\section{APPENDIX C}

Proof of Lemma $7(\Rightarrow)$ Suppose that $\mathbf{P Q}=\mathbf{Q P}$. Then

$$
\begin{aligned}
\mathbf{P Q} & -\mathbf{Q P} \\
& =\left(\mathbf{B}_{P} \otimes \mathbf{I}_{p_{1}}^{r}\right)\left(\mathbf{B}_{Q} \otimes \mathbf{I}_{q_{1}}^{r}\right)-\left(\mathbf{B}_{Q} \otimes \mathbf{I}_{q_{1}}^{r}\right)\left(\mathbf{B}_{P} \otimes \mathbf{I}_{p_{1}}^{r}\right) \\
& =\mathbf{B}_{P} \mathbf{B}_{Q} \otimes \mathbf{I}_{p_{1}}^{r} \mathbf{I}_{q_{1}}^{r}-\mathbf{B}_{Q} \mathbf{B}_{P} \otimes \mathbf{I}_{q_{1}}^{r} \mathbf{I}_{p_{1}}^{r} \\
& =\left(\mathbf{B}_{P} \mathbf{B}_{Q}-\mathbf{B}_{Q} \mathbf{B}_{P}\right) \otimes \mathbf{I}_{\left(p_{1}+q_{1}\right) \bmod r}^{r}=\mathbf{0},
\end{aligned}
$$

which implies that $\mathbf{B}_{P} \mathbf{B}_{Q}=\mathbf{B}_{Q} \mathbf{B}_{P}$ because $\mathbf{I}_{\left(p_{1}+q_{1}\right) \bmod r}^{r} \neq \mathbf{0}$.

$$
\begin{aligned}
& (\Leftarrow) \text { If } \mathbf{B}_{P} \mathbf{B}_{Q}=\mathbf{B}_{Q} \mathbf{B}_{P} \text {, then } \\
& \begin{aligned}
\mathbf{P Q} & =\mathbf{B}_{P} \mathbf{B}_{Q} \otimes \mathbf{I}_{\left(p_{1}+q_{1}\right) \bmod r}^{r} \\
& =\mathbf{B}_{Q} \mathbf{B}_{P} \otimes \mathbf{I}_{\left(q_{1}+p_{1}\right) \bmod r}^{r}=\mathbf{Q P} .
\end{aligned}
\end{aligned}
$$

\section{APPENDIX D}

Example 11. Consider the $2 \times 3$ all-ones base matrix $B$. Suppose that B is lifted twice to form the QC-LDPC paritycheck matrix

$$
\mathbf{H}=\mathbf{B}^{\uparrow m \circlearrowleft r}=\left[\begin{array}{lll}
\mathbf{H}_{1,1} & \mathbf{H}_{1,2} & \mathbf{H}_{1,3} \\
\mathbf{H}_{2,1} & \mathbf{H}_{2,2} & \mathbf{H}_{2,3}
\end{array}\right]_{2 m r \times 3 m r},
$$

where the circulant-block permutation matrices $\mathbf{H}_{i, j}$ are all non-overlapping. After row and column permutations, $\mathbf{H}$ can be re-written as

$$
\mathbf{H}=\left[\begin{array}{ccc}
\mathbf{I}_{0}^{m r} & \mathbf{I}_{0}^{m r} & \mathbf{I}_{0}^{m r} \\
\mathbf{I}_{0}^{m r} & \mathbf{P} & \mathbf{Q}
\end{array}\right],
$$

where $\mathbf{P}$ and $\mathbf{Q}$ are circulant-block. (This re-writing of $\mathbf{H}$ is not necessary, but it simplifies the following arguments.) Now consider the $3 m r$-tuple

$$
\mathbf{c}^{\top}=\left[(\mathbf{P}+\mathbf{Q}) \mathbf{x} ; \quad\left(\mathbf{I}_{0}^{m r}+\mathbf{Q}\right) \mathbf{x} ; \quad\left(\mathbf{I}_{0}^{m r}+\mathbf{P}\right) \mathbf{x}\right],
$$

where $\mathbf{x}$ is a arbitrary weight one column vector and ";" is used to denote stacking of column vectors. Since the permutation matrices comprising $\mathbf{H}$ are non-overlapping, the Hamming weight of $\mathbf{c}$ is $\operatorname{wt}(\mathbf{c})=(2+1) !=6$. Then

$$
\begin{aligned}
\mathbf{s}^{\top} & =\mathbf{H} \cdot \mathbf{c}^{\top}\left[\begin{array}{c}
\left(\mathbf{P}+\mathbf{Q}+\mathbf{I}_{0}^{m r}+\mathbf{Q}+\mathbf{I}_{0}^{m r}+\mathbf{P}\right) \mathbf{x} \\
(\mathbf{P}+\mathbf{Q}+\mathbf{P}+\mathbf{P Q}+\mathbf{Q}+\mathbf{Q P}) \mathbf{x}
\end{array}\right] \\
& =\left[\begin{array}{c}
\mathbf{0} \\
(\mathbf{P Q}+\mathbf{Q P}) \mathbf{x}
\end{array}\right],
\end{aligned}
$$

and $\mathbf{c}$ is a codeword if and only if $(\mathbf{P Q}+\mathbf{Q P}) \mathbf{x}=\mathbf{0}$. Consequently, if PQ and QP have an overlapping column, i.e., if $\mathbf{P}$ and $\mathbf{Q}$ are not strongly noncommutative, then there exists an $\mathbf{x}$ such that $\mathbf{c}$ is a codeword. If $\mathbf{P}$ and $\mathbf{Q}$ are strongly noncommutative, then $(\mathbf{P Q}+\mathbf{Q P}) \mathbf{x} \neq \mathbf{0}$ and $\mathbf{c}$ corresponds to a $(6, f)$ near-codeword, where the Hamming weight of the syndrome vector wt $(\mathbf{s})=f$ denotes the number of unsatisfied parity-check equations.

\section{REFERENCES}

[1] J. Thorpe, "Low-density parity-check (LDPC) codes constructed from protographs," Jet Propulsion Laboratory, Pasadena, CA, INP Progress Report 42-154, Aug. 2003.

[2] R. M. Tanner, "A recursive approach to low complexity codes," IEEE Transactions on Information Theory, vol. 27, no. 5, pp. 533-547, Sept. 1981.

[3] W. S. Massey, Algebraic Topology: an Introduction. New York: Springer-Verlag, Graduate Texts in Mathematics, Vol. 56, 1977.

[4] R. G. Gallager, "Low-density parity-check codes," IRE Transactions on Information Theory, vol. 8, no. 1, pp. 21-28, Jan. 1962.

[5] T. J. Richardson and R. L. Urbanke, "Multi-edge type LDPC codes," in Workshop honouring Prof. Bob McEliece on his 60th birthday, Pasadena, CA, May 2002.

[6] D. Divsalar, S. Dolinar, C. Jones, and K. Andrews, "Capacityapproaching protograph codes," IEEE Journal on Selected Areas in Communications, vol. 27, no. 6, pp. 876-888, Aug. 2009. 
[7] A. Abbasfar, D. Divsalar, and K. Yao, "Accumulate repeat accumulate codes," IEEE Transactions on Communications, vol. 55, no. 4, pp. 692702, Apr. 2007.

[8] D. G. M. Mitchell, A. E. Pusane, and D. J. Costello, Jr., "Minimum distance and trapping set analysis of protograph-based LDPC convolutional codes," IEEE Transactions on Information Theory, vol. 59, no. 1, pp. 254-281, Jan. 2013.

[9] Y. Kou, S. Lin, and M. P. C. Fossorier, "Low-density parity-check codes based on finite geometries: a rediscovery and new results," IEEE Transactions on Information Theory, vol. 47, no. 7, pp. 2711-2736, Nov. 2001.

[10] M. P. C. Fossorier, "Quasi-cyclic low-density parity-check codes from circulant permutation matrices," IEEE Transactions on Information Theory, vol. 50, no. 8, pp. 1788-1793, Aug. 2004.

[11] H. Tang, J. Xu, S. Lin, and K. A. S. Abdel-Ghaffar, "Codes on finite geometries," IEEE Transactions on Information Theory, vol. 51, no. 7, pp. 572-596, Nov. 2005.

[12] S. Myung, K. Yang, and J. Kim, "Quasi-cyclic LDPC codes for fast encoding," IEEE Transactions on Information Theory, vol. 51, no. 8, pp. 2894-2901, Aug. 2005.

[13] S. Myung, K. Yang, and Y. Kim, "Lifting methods for quasi-cyclic LDPC codes," IEEE Communications Letters, vol. 10, no. 6, pp. 489491, June 2006.

[14] Z. Li, L. Chen, L. Zeng, S. Lin, and W. H. Fong, "Efficient encoding of quasi-cyclic low-density parity-check codes," IEEE Transactions on Communications, vol. 54, no. 1, pp. 71-81, Jan. 2006.

[15] S. Song, B. Zhou, S. Lin, and K. Abdel-Ghaffar, "A unified approach to the construction of binary and nonbinary quasi-cyclic LDPC codes based on finite fields," IEEE Transactions on Communications, vol. 57 no. 1, pp. 84-93, Jan 2009.

[16] J. Kang, Q. Huang, L. Zhang, B. Zhou, and S. Lin, "Quasi-cyclic LDPC codes: an algebraic construction," IEEE Transactions on Communications, vol. 58, no. 5, pp. 1383-1396, May 2010.

[17] L. Zhang, Q. Huang, S. Lin, K. Abdel-Ghaffar, and I. F. Blake, "Quasicyclic LDPC codes: An algebraic construction, rank analysis, and codes on latin squares," IEEE Transactions on Communications, vol. 58, no. 11 , pp. 3126-3139, Nov. 2010.

[18] L. Zhang, S. Lin, K. Abdel-Ghaffar, Z. Ding, and B. Zhou, "Quasi-cyclic LDPC codes on cyclic subgroups of finite fields," IEEE Transactions on Communications, vol. 59, no. 9, pp. 2330-2336, Sept. 2011.

[19] L. Chen, J. Xu, I. Djurdjevic, and S. Lin, "Near-Shannon-limit quasicyclic low-density parity-check codes," IEEE Transactions on Communications, vol. 52, no. 7, pp. 1038-1042, July 2004.

[20] R. M. Tanner, D. Sridhara, A. Sridharan, T. E. Fuja, and D. J. Costello, Jr., "LDPC block and convolutional codes based on circulant matrices," IEEE Transactions on Information Theory, vol. 50, no. 12, pp. 29662984, Dec. 2004.

[21] R. Smarandache and P. O. Vontobel, "Quasi-cyclic LDPC codes: Influence of proto- and Tanner-graph structure on minimum Hamming distance upper bounds," IEEE Transactions on Information Theory, vol. 58, no. 2, pp. 585-607, Feb. 2012.

[22] I. E. Bocharova, F. Hug, R. Johannesson, B. D. Kudryashov, and R. V. Satyukov, "Searching for voltage graph-based LDPC tailbiting codes with large girth," IEEE Transactions on Information Theory, vol. 58, no. 4, pp. 2265-2279, Apr. 2012.

[23] Z. Wang and Z. Cui, "Low-complexity high-speed decoder design for quasi-cyclic LDPC codes," IEEE Transactions on Very Large Scale Integration (VLSI) Systems, vol. 15, no. 1, pp. 104-114, Jan. 2007.

[24] Y. Dai, Z. Yan, and N. Chen, "Memory-efficient and high-throughput decoding of quasi-cyclic LDPC codes," IEEE Transactions on Communications, vol. 57, no. 4, pp. 879-883, Apr. 2009.

[25] D. J. C. MacKay and M. C. Davey, "Evaluation of Gallager codes for short block length and high rate applications," in IMA Volumes in Mathematics and its Applications, Vol. 123: Codes, Systems, and Graphical Models. Springer-Verlag, 2001, pp. 113-130.

[26] M. E. O'Sullivan, "Algebraic construction of sparse matrices with large girth," IEEE Transactions on Information Theory, vol. 52, no. 2, pp. 718-727, Feb. 2006

[27] S. Kim, J.-S. No, H. Chung, and D.-J. Shin, "Quasi-cyclic low-density parity-check codes with girth larger than 12," IEEE Transactions on Information Theory, vol. 53, no. 8, pp. 2885-2891, Aug. 2007.

[28] N. Bonello, S. Chen, and L. Hanzo, "Construction of regular quasicyclic protograph LDPC codes based on Vandermonde matrices," IEEE Transactions on Vehicular Technology, vol. 57, no. 4, pp. 2583-2588, July 2008

[29] H. Park, S. Hong, J.-S. No, and D.-J. Shin, "Design of multiple-edge protographs for QC LDPC codes avoiding short inevitable cycles," IEEE
Transactions on Information Theory, vol. 59, no. 7, pp. 4598-4614, July 2013.

[30] R. Asvadi, A. H. Banihashemi, and M. Ahmadian-Attari, "Lowering the error floor of LDPC codes using cyclic liftings," IEEE Transactions on Information Theory, vol. 57, no. 4, pp. 2213-2224, Apr. 2011.

[31] M. Karimi and A. H. Banihashemi, "Counting short cycles of quasi cyclic protograph LDPC codes," IEEE Communications Letters, vol. 16, no. 3, pp. 400-403, Mar. 2012.

[32] B. K. Butler and P. H. Siegel, "Bounds on the minimum distance of punctured quasi-cyclic LDPC codes," IEEE Transactions on Information Theory, vol. 59, no. 7, p. 4584, July 2013.

[33] Low Density Parity Check Codes for Use in Near-earth and Deep Space Applications, The Consultative Committee for Space Data Systems Std., Sept. 2007. [Online]. Available: http://public.ccsds.org/publications/OrangeBooks.aspx

[34] Y. Wang, S. Draper, and J. Yedida, "Hierarchical and high-girth QC LDPC codes," IEEE Transactions on Information Theory, vol. 59, no. 7, pp. 4553-4583, July 2013.

[35] L. Wei, "Several properties of short LDPC codes," IEEE Transactions on Communications, vol. 52, no. 5, pp. 721-727, May 2004.

[36] R. Smarandache, D. G. M. Mitchell, and D. J. Costello, Jr., "Partially quasi-cyclic protograph-based LDPC codes," in Proc. IEEE International Conference on Communications, Kyoto, Japan, June 2011.

[37] X.-Y. Hu, E. Eleftheriou, and D. M. Arnold, "Regular and irregular progressive edge-growth Tanner graphs," IEEE Transactions on Information Theory, vol. 51, no. 1, pp. 386-398, Jan. 2005.

[38] I. E. Bocharova, B. D. Kudryashov, and R. V. Satyukov, "Graphbased convolutional and block LDPC codes," Problems of Information Transmission, vol. 45, no. 4, pp. 357-377, 2009

[39] W. Bosma, J. Cannon, and C. Playoust, "The Magma algebra system. I. The user language," J. Symbolic Comput., vol. 24, no. 3-4, pp. 235-265, 1997, computational algebra and number theory (London, 1993). [Online]. Available: http://dx.doi.org/10.1006/jsco.1996.0125

[40] M. G. Luby, M. Mitzenmacher, M. A. Shokrollahi, and D. A. Spielman, "Improved low-density parity-check codes using irregular graphs," IEEE Transactions on Information Theory, vol. 47, no. 2, pp. 585-598, Feb. 2001.

[41] J. Xu, L. Chen, I. Djurdjevic, S. Lin, and K. A. S. Abdel-Ghaffar, "Construction of regular and irregular LDPC codes: Geometry decomposition and masking," IEEE Transactions on Information Theory, vol. 54, no. 1, pp. 121-134, Jan. 2007.

[42] C. Di, T. J. Richardson, and R. L. Urbanke, "Weight distribution of lowdensity parity-check codes," IEEE Transactions on Information Theory, vol. 52, no. 11, pp. 4839-4855, Nov. 2006.

[43] T. J. Richardson, M. A. Shokrollahi, and R. L. Urbanke, "Design of capacity approaching irregular low-density parity-check codes," IEEE Transactions on Information Theory, vol. 47, no. 2, pp. 619-637, Feb. 2001.

[44] S.-Y. Chung, "On the construction of some capacity-approaching coding schemes," Ph.D. dissertation, Massachusetts Institute of Technology, Cambridge, MA, Sept. 2000.

[45] Air Interface for Fixed and Mobile Broadband Wireless Access Systems IEEE P802.16e/D12, Std., Oct. 2005. 